Non-periodic homogenization of infinitesimal strain plasticity equations

\author{
Martin Heida, Ben Schweizer
}




\title{
Non-periodic homogenization of infinitesimal strain plasticity equations
}

\author{
Martin Heida and Ben Schweizer*
}

May 21, 2014

\begin{abstract}
We consider the Prandtl-Reuss model of plasticity with kinematic hardening, aiming at a homogenization result. For a sequence of coefficient fields and corresponding solutions $u^{\varepsilon}$, we ask whether we can characterize weak limits $u$ when $u^{\varepsilon} \rightarrow u$ as $\varepsilon \rightarrow 0$. We assume neither periodicity nor stochasticity for the coefficients, but we demand an abtract averaging property of the homogeneous system on reference volumes. Our conclusion is an effective equation on general domains with general right hand sides. The effective equation uses a causal evolution operator $\Sigma$ that maps strains to stresses; more precisely, in each spatial point $x$, given the evolution of the strain in the point $x$, the operator $\Sigma$ provides the evolution of the stress in $x$.
\end{abstract}

Keywords: small strain plasticity, non-periodic homogenization

\section{Introduction}

Due to its relevance in applications, the homogenization of plasticity models has become a very active field of research. While the homogenization of other equations of continuum mechanics is meanwhile well established (e.g. elasticity models for solids or Navier-Stokes equations for fluids), the homogenization of plasticity models is more involved. Only recently results have been obtained, and, so far, they concern only the periodic setting (with the exception of [18], which is restricted to one space dimension). Plasticity imposes new analytical challenges, the most relevant is probably the lack of spatial regularity due to the singular character of the flow rule.

The current homogenization literature on plasticity is mainly concerned with infinitesimal strain plasticity and an additive decomposition of the strain tensor (for an exception see [12] and the references therein). With the single exception of [8], all contributions include an hardening effect. We also include hardening by assuming that the tensor $B^{\varepsilon}$ below is positive definite. We write the plasticity system (we refer to $[1,13]$ for modelling aspects) in the form

$$
\begin{aligned}
-\nabla \cdot \sigma^{\varepsilon} & =f, & \sigma^{\varepsilon} & =C_{\varepsilon}^{-1} e^{\varepsilon}, \\
\nabla^{s} u^{\varepsilon} & =e^{\varepsilon}+p^{\varepsilon}, & \partial_{t} p^{\varepsilon} & \in \partial \Psi_{\varepsilon}\left(\sigma^{\varepsilon}-B_{\varepsilon} p^{\varepsilon}\right) .
\end{aligned}
$$

${ }^{*}$ Technische Universität Dortmund, Fakultät für Mathematik, Vogelpothsweg 87, D-44227 Dortmund, Germany. 
The equations are posed on a space-time cylinder $Q \times(0, T)$, where $Q \subset \mathbb{R}^{n}$ is a bounded Lipschitz domain. The equations (1.1) are accompanied by initial and boundary conditions, the unknowns are the deformation $u^{\varepsilon}$, the elastic strain $e^{\varepsilon}$, the plastic strain $p^{\varepsilon}$, and the stress $\sigma^{\varepsilon}$. Given are a right hand side $f$, the coefficient tensors $C_{\varepsilon}$ and $B_{\varepsilon}$, and the convex function $\Psi_{\varepsilon}$ that describes the flow rule.

The parameter $\varepsilon>0$ indicates that we are given a family of coefficient fields. Our interest is to investigate the corresponding solutions $u^{\varepsilon}$ and to characterize weak limits $u$ of a sequence of solutions. This is the principle goal in homogenization theory. So far, only the (essentially) periodic case has been studied, i.e.coefficients of the form $C_{\varepsilon}(x)=C_{1}(x, x / \varepsilon)$, and similarly for $B_{\varepsilon}, \Psi_{\varepsilon}$.

We present a result that is obtained with the needle-problem approach to homogenization, an approach that was introduced with [20]. In this approach, mathematical homogenization is regarded as a two-step procedure: Step 1 treats cell-problems, concluding that, on a representative elementary volume (REV), the material behaves in a well-defined way: an input (e.g. the averaged gradient $\xi$ of the solution) results in a certain output (e.g. the averaged flux $A^{*} \xi$ ). Step 2 treats arbitrary right hand sides $f$ and arbitrary domains $Q$. The conclusion of Step 2 is: the material law (obtained from the REV) characterizes the behavior of the material on the macroscopic scale.

The needle-problem approach is concerned with Step 2 of this program. We assume that the material parameters allow averaging: for solutions on simplices, corresponding to affine boundary data $\xi$ and vanishing forces $f=0$, averages of the stress converge weakly to some deterministic quantity $\Sigma(\xi)$. We then conclude that the map $\Sigma$ characterizes solutions on general domains $Q$, with general boundary data and with general forces.

The result of Step 2 is relevant as such, since it justifies to extract effective coefficients from REV-tests. On the other hand, in a more specific setting (e.g. a problem with stochastic coefficients), one may be in a position to verify the averaging assumption (Step 1); once this is done, the needle-problem result allows to conclude the full homogenization limit.

\section{$1.1 \quad$ Literature}

Homogenization theory has become a well-established part of the theory of partial differential equations. The most relevant approaches are energy methods, two-scale convergence methods, and periodic unfolding. Having at disposal a well-developed theory for linear equations with periodic coefficients, much of the more recent research is focussed on stochastic homogenization $[11,18,22]$ and nonlinear problems $[5,7,9$, $16,25]$.

The homogenization of the highly nonlinear plasticity systems is quite recent. A first mathematical formulation of the effective two-scale limit system (for a periodic plasticity system with hardening) seems to appear in [2] (we refer to Subsection 1.4 below for a description of the limit system). The rigorous derivation of the effective two-scale system was obtained with various approaches, covering a varying generality of the coefficient functions. A consequent use of variational aspects and the method of two-scale convergence was exploited first by Visintin, see [23, 24, 26], and later in [21]. Alber and Nesenenko use the method of two-scale convergence and combine it with phase-shift convergence to obtain rigorous results, see [3, 17]. A homogenization proof 
based on Tartar's energy method is presented in [19], and general homogenization results for rate-independent systems have been developed in [15, 16]. The recent analysis of [21] is entirely based on two-scale convergence, it allows quite general monotone flow rules and it clearifies the necessary conditions regarding $x$ - and $y$ dependence of the flow function; we refer to this work also for a further description of the literature.

\subsection{Setting of the problem}

We consider the $\varepsilon$-Problem (1.1) on a bounded Lipschitz domain $Q \subset \mathbb{R}^{n}, n \leq 3$. More precisely, for some time horizon $T>0$, we consider solutions of the system (1.1) on $Q \times(0, T)$. For a fixed sequence $\varepsilon=\varepsilon_{j} \rightarrow 0$ we assume that a family of coefficients and coefficient functions $\left(C_{\varepsilon}, B_{\varepsilon}, \Psi_{\varepsilon}\right)$ is given.

The other data are the right hand side $f$ and the boundary data $w$. We complement system (1.1) with the boundary condition $u^{\varepsilon}=w$ on $\partial Q \times(0, T)$. We assume the regularity

$$
w \in H^{1}\left(0, T ; H^{1}\left(Q ; \mathbb{R}^{n}\right)\right), \quad f \in H^{1}\left(0, T ; L^{2}(Q)\right) .
$$

We restrict ourselves to the initial condition

$$
p^{\varepsilon}(0)=0 \text {. }
$$

Regarding the tensor fields $C_{\varepsilon}, B_{\varepsilon} \in L^{\infty}\left(Q ; \mathcal{L}\left(\mathbb{R}_{s}^{n \times n}, \mathbb{R}_{s}^{n \times n}\right)\right)$, where $\mathbb{R}_{s}^{n \times n}$ denotes the symmetric $n \times n$-matrices, we assume the boundedness and positivity

$$
\gamma|\xi|^{2} \leq \xi:\left(C_{\varepsilon}(x) \xi\right) \leq \frac{1}{\gamma}|\xi|^{2}, \quad \beta|\xi|^{2} \leq \xi:\left(B_{\varepsilon}(x) \xi\right) \leq \frac{1}{\beta}|\xi|^{2}
$$

for all $\xi \in \mathbb{R}_{s}^{n \times n}$ and a.e. $x \in Q$, where the constants $\gamma, \beta>0$ do not depend on $\varepsilon$. We furthermore assume that $C_{\varepsilon}$ and $B_{\varepsilon}$ are symmetric with respect to the scalar product on $\mathbb{R}_{s}^{n \times n}$. We finally assume that $\Psi_{\varepsilon}(., x): \mathbb{R}_{s}^{n \times n} \rightarrow \overline{\mathbb{R}}$ is convex, non-negative, and that the convex conjugate function $\Psi_{\varepsilon}^{*}$ satisfies a Lipschitz continuity condition: For $C=C(\varepsilon)>0$ holds

$$
\left|\Psi_{\varepsilon}^{*}\left(\sigma ; x_{1}\right)-\Psi_{\varepsilon}^{*}\left(\sigma ; x_{2}\right)\right| \leq C(\varepsilon)\left|x_{1}-x_{2}\right||\sigma| .
$$

Given coefficients $C_{\varepsilon}$ and $B_{\varepsilon}$, we use the notation

$$
|\xi|_{C_{\varepsilon}}^{2}:=\xi:\left(C_{\varepsilon}(x) \xi\right), \quad|\xi|_{B_{\varepsilon}}^{2}:=\xi:\left(B_{\varepsilon}(x) \xi\right) .
$$

Due to (1.4), the norms $|\cdot|_{C_{\varepsilon}}$ and $|\cdot|_{B_{\varepsilon}}$ are equivalent to the Euclidean norm on $\mathbb{R}_{s}^{n \times n}$.

Remark 1. No initial conditions for $u^{\varepsilon}, e^{\varepsilon}$ and $\sigma^{\varepsilon}$ are imposed. The initial data (1.3) imply: $C_{\varepsilon} \sigma^{\varepsilon}(0)=\nabla^{s} u^{\varepsilon}(0)$, hence $(1.1)_{1}$ is equivalent to

$$
-\nabla \cdot\left(C_{\varepsilon}^{-1} \nabla^{s} u^{\varepsilon}(0)\right)=f(0) .
$$

Due to positivity of $C_{\varepsilon}$ in the space of symetric matrices, the elasticity system (1.6) posesses a unique solution $u^{\varepsilon}(0)$ to the boundary condition $\left.u^{\varepsilon}(0)\right|_{\partial Q}=\left.w(0)\right|_{\partial Q}$. This solution also provides $e^{\varepsilon}(0)=\nabla^{s} u^{\varepsilon}(0)$ and $\sigma^{\varepsilon}(0)=C_{\varepsilon}^{-1} e^{\varepsilon}(0)$. 
Definition 1.1 (Weak solution to the $\varepsilon$-problem). We say that $\left(u^{\varepsilon}, e^{\varepsilon}, p^{\varepsilon}, \sigma^{\varepsilon}\right)$ is a weak solution to the $\varepsilon$-problem (1.1) on $Q$ with boundary data $w$ if the following holds: $u^{\varepsilon}=v^{\varepsilon}+w$ with

$$
v^{\varepsilon} \in H^{1}\left(0, T ; H_{0}^{1}(Q)\right), \quad e^{\varepsilon}, p^{\varepsilon}, \sigma^{\varepsilon} \in H^{1}\left(0, T ; L^{2}\left(Q ; \mathbb{R}_{s}^{n \times n}\right)\right),
$$

for every $\varphi \in L^{2}\left(0, T ; H_{0}^{1}(Q)\right)$ holds

$$
\int_{0}^{T} \int_{Q} \sigma^{\varepsilon}: \nabla^{s} \varphi=\int_{0}^{T} \int_{Q} f \cdot \varphi
$$

and the relations

$$
\partial_{t} p^{\varepsilon} \in \partial \Psi_{\varepsilon}\left(\sigma^{\varepsilon}-B_{\varepsilon} p^{\varepsilon}\right), \quad \nabla^{s} u^{\varepsilon}=e^{\varepsilon}+p^{\varepsilon}, \quad \sigma^{\varepsilon}=C_{\varepsilon} e^{\varepsilon}
$$

hold pointwise almost everywhere on $Q \times(0, T)$.

For fixed $Q$ and $T$, we denote the two time dependent function spaces of (1.7) as $\mathcal{V}_{0}^{1}:=H^{1}\left(0, T ; L^{2}\left(Q ; \mathbb{R}_{s}^{n \times n}\right)\right)$ and $\mathcal{V}_{1}^{1}:=H^{1}\left(0, T ; H_{0}^{1}(Q)\right)$. The upper index shows the regularity in time, the lower index shows the regularity in space (using the fact that $H^{0} \equiv L^{2}$ ). We emphasize that the indices express the regularity, but also the number of components is different in the two spaces.

Theorem 1.2 (Existence of weak solutions). Let the data be as in (1.2)-(1.5). Then, for every $\varepsilon>0$, there exists a unique weak solution $\left(u^{\varepsilon}, e^{\varepsilon}, p^{\varepsilon}, \sigma^{\varepsilon}\right)$ to (1.1) in the sense of Definition 1.1. The solution satisfies the a priori estimate

$$
\left\|u^{\varepsilon}\right\|_{\mathcal{V}_{1}^{1}}+\left\|e^{\varepsilon}\right\|_{\mathcal{V}_{0}^{1}}+\left\|p^{\varepsilon}\right\|_{\mathcal{V}_{0}^{1}}+\left\|\sigma^{\varepsilon}\right\|_{\mathcal{V}_{0}^{1}} \leq C
$$

where $C=C(w, f, \beta, \gamma)$ is independent of $\varepsilon>0$.

Similar existence results are available in the literature. We note that Theorem 1.1 of [19] cannot be applied, since we do not have the term $\partial_{t}^{2} u$ in (1.1), while such an inertia term is included [19]. The proof relies on the a priori estimate (1.10), which is shown in the appendix.

\subsection{Main Result}

Our main result is a homogenization result. We regard it as a justification of the concept of representative elementary volumes: We assume that the system has an averaging property on standard elements (simplices) with standard data (affine boundary data and vanishing right hand side), see Definition 1.3. We conclude from the averaging property the homogenization result for general data, see Theorem 1.6.

We next want to formulate the averaging property. Since plasticity equations include hysteresis effects, the evolution of the average stress in a representative volume will not only depend on the applied strain, but it will depend on the whole history of the strain. We must therefore work with operators that map a strain history to a stress history,

$$
\Sigma: H^{1}\left(0, T ; \mathbb{R}_{s}^{n \times n}\right) \rightarrow H^{1}\left(0, T ; \mathbb{R}_{s}^{n \times n}\right) .
$$

We emphasize that no spatial dependence appears in this operator. 
We call an operator $\Sigma$ as in (1.11) a causal operator, if and only if, for every $t \in[0, T]$, the value $\Sigma(\xi, t):=\Sigma(\xi)(t)$ is independent of $\left.\xi\right|_{(t, T]}$ in the sense that

$$
\xi_{1}, \xi_{2} \in H^{1}\left(0, T ; \mathbb{R}_{s}^{n \times n}\right) \quad \text { with }\left.\quad \xi_{1}\right|_{[0, t)}=\left.\xi_{2}\right|_{[0, t)} \quad \Rightarrow \quad \Sigma\left(\xi_{1}, t\right)=\Sigma\left(\xi_{2}, t\right) .
$$

We can now formulate the averaging property for plasticity equations. We use $f_{\mathcal{T}} f:=$ $|\mathcal{T}|^{-1} \int_{\mathcal{T}} f$ to denote the average of a function $f$ on $\mathcal{T}$

Definition 1.3 (Averaging property). For fixed $T>0$ we say that the plasticity system with coefficients $C_{\varepsilon}, B_{\varepsilon}$ and $\Psi_{\varepsilon}$ allows averaging if and only if there exist two causal operators $\Sigma$ and $\Pi$ with the following property.

Let $\mathcal{T} \subset \mathbb{R}^{n}$ be any simplex and let $\xi \in H^{1}\left(0, T ; \mathbb{R}_{s}^{n \times n}\right)$ and $a \in H^{1}\left(0, T ; \mathbb{R}^{n}\right)$ define affine (time dependent) boundary data $w(x, t)=\xi(t) \cdot x+a(t)$. Let $\left(u^{\varepsilon}, e^{\varepsilon}, p^{\varepsilon}, \sigma^{\varepsilon}\right)$ be the solution to the $\varepsilon$-problem with $f=0$ and boundary data $w$. Then, as $\varepsilon \rightarrow 0$, for almost every $t \in(0, T)$, averages of stress and strain convergence:

$$
f_{\mathcal{T}} p^{\varepsilon}(t) \rightarrow \Pi(\xi, t), \quad f_{\mathcal{T}} \sigma^{\varepsilon}(t) \rightarrow \Sigma(\xi, t) .
$$

Remark 2. The averaging property of Definition 1.3 is independent of a in the following sense: if the averaging property holds for $a \equiv 0$ with solution $\left(u^{\varepsilon}, e^{\varepsilon}, p^{\varepsilon}, \sigma^{\varepsilon}\right)$, then it holds for arbitrary $a \in H^{1}\left(0, T ; \mathbb{R}^{n}\right)$ with solution $\left(u^{\varepsilon}+a, e^{\varepsilon}, p^{\varepsilon}, \sigma^{\varepsilon}\right)$.

Remark 3. The averaging property of Definition 1.3 implies the following averaging property for the elasticity system (which is a classical homogenization result): There exists an effective tensor $C_{*}$ such that, given $\xi \in \mathbb{R}^{n \times n}$ and $a \in \mathbb{R}^{n}$, the solutions $u^{\varepsilon}$ of the problem

$$
-\nabla \cdot\left(C_{\varepsilon}^{-1} \nabla^{s} u^{\varepsilon}\right)=0 \quad \text { in } \mathcal{T}, \quad u^{\varepsilon}(x)=\xi \cdot x+a \quad \text { on } \partial \mathcal{T}
$$

satisfy the convergence of the average stress

$$
\lim _{\varepsilon \rightarrow 0} f_{\mathcal{T}} C_{\varepsilon}^{-1} \nabla^{s} u^{\varepsilon}=C_{*}^{-1} \xi
$$

This property can be concluded by considering $t=0$ in the sense of traces in (1.12). The map $\xi \mapsto \Sigma(\xi, 0)$ (considering $\xi$ on the right hand side as the constant function in time) is necessarily linear and hence given by some tensor $C_{*}^{-1} \in \mathcal{L}\left(\mathbb{R}_{s}^{n \times n}, \mathbb{R}_{s}^{n \times n}\right)$. The positivity of $C_{*}^{-1}$ can be concluded a posteriori from the elasticity system.

Remark 4. The Gauß theorem implies that, for almost every $t \in(0, T)$, the matrix $\xi(t)$ is the average of $\nabla^{s} u^{\varepsilon}(., t)$ on $\mathcal{T}$. From this and the decomposition $\nabla^{s} u=e^{\varepsilon}+p^{\varepsilon}$, we obtain with the operator $E:=\mathrm{Id}-\Pi$

$$
f_{\mathcal{T}} \nabla^{s} u^{\varepsilon}(t) \rightarrow \xi(t) \quad \text { and } \quad f_{\mathcal{T}} e^{\varepsilon}(t) \rightarrow E(\xi, t)
$$

Regarding the further mathematical analysis, we remark that we could as well drop the convergence condition on $f_{\mathcal{T}} p^{\varepsilon}(t)$ in Definition 1.3. Our main homogenization result holds also without the convergence property of the $p^{\varepsilon}$-averages. 
Definition 1.4 (Effective problem). The effective plasticity problem is given by the momentum equation

$$
-\nabla \cdot \Sigma\left(\nabla^{s} u\right)=f .
$$

We say that $u$ is a weak solution to the effective problem (1.13) with boundary condition $w \in H^{1}\left(0, T ; H^{1}\left(Q ; \mathbb{R}^{n}\right)\right)$, if $u$ can be written as $u=w+v$ for $v \in H^{1}\left(0, T ; H_{0}^{1}\left(Q ; \mathbb{R}^{n}\right)\right)$, if the regularity $\Sigma\left(\nabla^{s} u\right) \in L^{2}(Q \times(0, T))$ holds and if (1.13) holds in the sense of distributions on $Q \times(0, T)$.

Regarding the solution concept of Definition 1.4 and, in particular, the expression $\Sigma\left(\nabla^{s} u\right)$, we note the following: We demand for solutions the regularity $\nabla^{s} u \in$ $H^{1}\left(0, T ; L^{2}\left(Q ; \mathbb{R}_{s}^{n \times n}\right)\right)$, hence $\nabla^{s} u(x,.) \in H^{1}\left(0, T ; \mathbb{R}_{s}^{n \times n}\right)$ for almost every $x \in Q$. As a consequence, the expression $\Sigma\left(\nabla^{s} u\right)(x, t)=\Sigma\left(\nabla^{s} u(x,), t.\right)$ is well-defined for almost every $x \in Q$ and almost every $t \in(0, T)$. In our solution concept, we demand that this function is of class $\Sigma\left(\nabla^{s} u\right) \in L^{2}(Q \times(0, T))$.

For our homogenization result, we will have to assume not only the averaging property, but also the following solvability property for $\Sigma$.

Definition 1.5 (Admissibility of $\Sigma$ ). We say that an operator $\Sigma: H^{1}\left(0, T ; \mathbb{R}_{s}^{n \times n}\right) \rightarrow$ $H^{1}\left(0, T ; \mathbb{R}_{s}^{n \times n}\right)$ is admissible, if, for every $Q, f$, and $w$ as above, the effective problem of Definition 1.4 has a solution.

Remark 5. Let $\Sigma$ be a causal operator with the following property: For every sequence of solutions $u_{h}$ of the discretized effective problem (4.13) with $u_{h} \rightarrow u$ in $H^{1}\left(0, T ; H^{1}(Q)\right)$ as $h \rightarrow 0$, there holds

$$
\Sigma\left(\nabla^{s} u_{h}\right) \rightarrow \Sigma\left(\nabla^{s} u\right) \text { weakly in } L^{2}\left(0, T ; L^{2}(Q)\right)
$$

Then $\Sigma$ is admissible in the sense of Definition 1.5.

Proof: Proposition 4.5 yields the well posedness of (4.13), the a priori estimate yields a bound for $u_{h}$ in $H^{1}\left(0, T ; H^{1}(Q)\right)$. We can therefore select a weakly convergent subsequence. Taking the limit $h \rightarrow 0$, exploiting (1.14), we obtain the solvability for the effective system.

Theorem 1.6 (Main Theorem). Let $Q \subset \mathbb{R}^{n}$ be open and bounded and let the data satisfy (1.2)-(1.5). Let the coefficients allow averaging in sense of Definition 1.3 with causal operators $\Sigma$ and $\Pi$. We assume that $\Sigma$ is admissible in the sense of Definition 1.5, i.e. that the effective problem posesses a solution. Then the following holds.

Let $\left(u^{\varepsilon}, e^{\varepsilon}, p^{\varepsilon}, \sigma^{\varepsilon}\right)$ be a sequence of solutions to the $\varepsilon$-problems (1.1) as specified in Definition 1.1. Then

$$
\begin{aligned}
& u^{\varepsilon} \rightarrow u \quad \text { weakly in } H^{1}\left(0, T ; H^{1}\left(Q ; \mathbb{R}^{n}\right)\right), \\
& p^{\varepsilon} \rightarrow \Pi\left(\nabla^{s} u\right), \quad \sigma^{\varepsilon} \rightarrow \Sigma\left(\nabla^{s} u\right) \quad \text { weakly in } H^{1}\left(0, T ; L^{2}\left(Q ; \mathbb{R}^{n \times n}\right)\right),
\end{aligned}
$$

where $u$ is the unique weak solution to the homogenized problem (1.13) with right hand side $f$ and boundary condition $w$ in the sense of Definition 1.4.

The proof of Theorem 1.6 is concluded in Subsection 4.4, an outline of the proof is given in Subsection 2.1. 


\subsection{Periodic Homogenization}

To make the connection to periodic homogenization, we recall results of [21], where the plasticity system

$$
\begin{aligned}
& \partial_{t}^{2} u^{\varepsilon}-\nabla \cdot \sigma^{\varepsilon}=f, \quad C_{\varepsilon} \sigma^{\varepsilon}=\nabla^{s} u^{\varepsilon}-p^{\varepsilon}, \\
& \partial_{t} p^{\varepsilon} \in g_{\varepsilon}\left(\sigma^{\varepsilon}-B_{\varepsilon} p^{\varepsilon}\right),
\end{aligned}
$$

is analyzed. We simplified here to a special case, omitting general internal variables. In our case, the monotone multi-valued map $g^{\varepsilon}$ is a subdifferential, $g^{\varepsilon}=\partial \Psi_{\varepsilon}$. With the unit cell $Y:=\left[0,1\left[^{n}\right.\right.$, periodic coefficients are constructed as

$$
C_{\varepsilon}(x):=C\left(x, \frac{x}{\varepsilon}\right), \quad B_{\varepsilon}(x):=B\left(x, \frac{x}{\varepsilon}\right), \quad g_{\varepsilon}(., x):=g\left(. ; x, \frac{x}{\varepsilon}\right) .
$$

In [21], the homogenization of (1.17)-(1.18) is performed and the following limit problem is established:

$$
\begin{aligned}
& \partial_{t}^{2} u-\nabla \cdot\left(\int_{Y} z d y\right)=f, \quad C z=\nabla_{x}^{s} u+\nabla_{y}^{s} v-w, \\
& \partial_{t} w \in g(z-B w ; x, y), \quad \nabla_{y} \cdot z=0 .
\end{aligned}
$$

Here, $z=z(x, y, t)$ is a two-scale stress variable, $w=w(x, y, t)$ a two-scale plastic strain variable, and $v=v(x, y, t)$ stands for fine-scale deformations, these are functions on $Q \times Y \times[0, T]$, while the strong limit $u=u(x, t)$ is a function on $Q \times[0, T]$. Theorem 2.2 of [21] yields, in particular, $u^{\varepsilon} \rightarrow u, \sigma^{\varepsilon} \rightarrow \int_{Y} z d y$ and $p^{\varepsilon} \rightarrow \int_{Y} w d y$.

We emphasize that we deal here with the quasi-static approximation, while in [21] the inertia term $\partial_{t}^{2} u^{\varepsilon}$ has been included. Nevertheless, let us formally investigate the form of the causal operator $\Sigma$ in this periodic setting. We assume that the coefficients do not depend on the macroscopic variable $x$.

Let $\xi \in H^{1}\left(0, T ; \mathbb{R}_{s}^{n \times n}\right)$ be a prescribed evolution of the macroscopic strain. Our aim is to characterize $\Sigma(\xi)$. We start with relations $(1.19)_{2}$ and $(1.20)_{2}$, from which we expect, since $\nabla_{x}^{s} u=\xi$ is independent of $y$,

$$
\xi-w_{\xi}=C z_{\xi}-\nabla_{y}^{s} v_{\xi}, \quad \nabla_{y} \cdot z_{\xi}=0 .
$$

In these two equations on $Y$, the functions are $w_{\xi}, z_{\xi} \in L^{2}\left(Y ; \mathbb{R}_{s}^{n \times n}\right), v_{\xi} \in H^{1}\left(Y ; \mathbb{R}^{n}\right)$, $\xi=\xi(t)$ is identified with a constant function on $Q$. We interpret the two equations as a form of a Helmholtz decomposition (which it is for $C=\mathrm{Id}$ ): Decomposing data $\xi-w_{\xi}$ into a solenoidal part and a gradient can determine the functions $z_{\xi}$ and $v_{\xi}$.

On this basis we consider, for every time instance $t$, the function $z_{\xi}(y)$ as determined by the data $\xi(t)$ and $w_{\xi}(y, t)$. We therefore regard $(1.20)_{1}$ as an equation for $w_{\xi}$,

$$
\partial_{t} w_{\xi}=g\left(z_{\xi}-B w_{\xi} ; y\right) \text {. }
$$

If this ordinary differential equation for the variable $w_{\xi}:[0, T] \rightarrow L^{2}\left(Y ; \mathbb{R}_{s}^{n \times n}\right)$ is solvable, we can expect $\Sigma(\xi):=\int_{Y} z_{\xi} d y$ and $\Pi(\xi):=\int_{Y} w_{\xi} d y$.

The relation $(1.19)_{1}$ can now be interpreted as the effective macroscopic system, and it corresponds to relation (1.13).

A similar perspective is used to perform stochastic homogenization, we present a result in [14]. The homogenization result follows (from Theorem 1.6), as soon as the properties of $\Sigma$ are verified in the stochastic setting. To show the averaging and the continuity properties, we construct the operator $\Sigma$ in analogy to the periodic case, sketched above. 


\section{The needle problem approach}

Our aim is to conclude from the averaging property (1.12) a homogenization result for the plasticity system (1.1). We will follow the principal idea of [20] and use the needle problem approach. This approach is based on a discretization of the domain (paramater $h>0$ ) and an auxiliary problem (the needle problem) with solution $u_{h}^{\varepsilon}$.

In the course of the proof, an appropriate discretization of the domain must be chosen and discretized equations must be formulated. We will deal with these more technical parts later and present first an outline of the proof.

\subsection{Outline of the proof of Theorem 1.6}

We construct a discretization of the domain. Given a sequence $u^{\varepsilon}$ of solutions, the triangulation is chosen in such a way that a compensated compactness property for the sequence $u^{\varepsilon}$ is available. The discretization of the domain allows to formulate the needle problem: We solve the original $\varepsilon$-system in each simplex of the triangulation, but impose the side condition that the solutions must be affine on the boundary of each simplex. The corresponding solutions are denoted as $u_{h}^{\varepsilon}$. We can now compare four functions: the original solution $u^{\varepsilon}$, the needle problem solution $u_{h}^{\varepsilon}$, the solution $u$ of the effective problem, and the solution $u_{h}$ of a discretized effective problem.

The four functions can be compared as indicated in scheme (2.1). The parameter of the small scale is $\varepsilon>0$, the parameter of the spatial discretization is $h>0$, where we only consider $\varepsilon \ll h$.

$$
\begin{array}{ccc}
u_{h}^{\varepsilon} & \stackrel{\text { Prop. } 4.5}{\longrightarrow} & u_{h} \\
\varepsilon, h \rightarrow 0 \uparrow \text { Prop. } 4.1 & & h \rightarrow 0 \downarrow \text { Prop. } 3.4 \\
u^{\varepsilon} & & u
\end{array}
$$

The scheme is justified as follows: The left double arrow (Proposition 4.1) indicates that $u_{h}^{\varepsilon}-u^{\varepsilon}$ is small for small parameters; this will essentially be obtained by testing the equations, exploiting the compensated compactness property for $u^{\varepsilon}$. The top arrow (Proposition 4.5) will be a consequence of the averaging property; we use that $u_{h}^{\varepsilon}$ has affine boundary data in every simplex. Finally, the right arrow (Proposition 3.4) is obtained with a finite element analysis of the limit problem.

We proceed as follows. In Subsection 2.2 we discuss the discretization of the domain with adapted grids. In Subsection 2.3 we introduce some function spaces, a discretization of the right hand side and a discretization of the boundary conditions. In Subsection 2.4, we introduce a spatially discretized version of the plasticity equations - the needle problem.

Section 3 is devoted to the study of the limit system. In particular, we show that solutions to the effective problem and to a spatial discretization of the effective problem are unique.

In Section 4, Theorem 1.6 is derived from three results on the auxiliary problems: $\lim _{h \rightarrow 0} \limsup _{\varepsilon \rightarrow 0}\left\|u^{\varepsilon}-u_{h}^{\varepsilon}\right\|_{L^{\infty}\left(0, T ; L^{2}(Q)\right)}=0$ is shown with energy methods in Proposition 4.1 and $u_{h}^{\varepsilon} \rightarrow u_{h}$ for $\varepsilon \rightarrow 0$ is shown with the averaging property in Proposition 
4.5. Using additionally the result $u_{h} \rightarrow u$ for $h \rightarrow 0$ of Proposition 3.4, we obtain our main result, $u^{\varepsilon} \rightarrow u$, i.e. Theorem 1.6.

\subsection{Adapted grids}

Let $Q \subset \mathbb{R}^{n}$ be a bounded domain in two or three space dimensions, $n \in\{2,3\}$. For arbitrary $h>0$, we approximate $Q$ with a polygonal domain $Q_{h} \subset Q$, the domain $Q_{h}$ is discretized with simplices in a triangulation $\mathbb{T}_{h}$. We demand

$\mathbb{T}_{h}:=\left\{\mathcal{T}_{k}\right\}_{k \in \Lambda_{h}} \quad$ is a triangulation of $Q_{h}, \quad \operatorname{diam}\left(\mathcal{T}_{k}\right)<h \quad \forall \mathcal{T}_{k} \in \mathbb{T}_{h}$,

$Q_{h}$ has the property that $x \in Q$, $\operatorname{dist}(x, \partial Q) \geq h$ implies $x \in Q_{h}$,

where $\mathcal{T}_{k}$ are disjoint open simplices and $\Lambda_{h} \subset \mathbb{N}$ is a finite set of indices. We always assume that the sequence of meshes is regular in the sense of [6], Section 3.1.

In [20], where the needle-problem approach was introduced, the following theorem is shown. It guarantees that, given a weakly convergent sequence $u^{\varepsilon} \in H^{1}(Q)$, we can always construct a polygonal domain $Q_{h}$ with a triangulation $\mathbb{T}_{h}$ as above such that additionally a compensated compactness property for the sequence $u^{\varepsilon}$ is satisfied.

Theorem 2.1 (Adapted grids and div-curl property, Theorem 1.3 in [20]). Let $Q \subset$ $\mathbb{R}^{n}, n \leq 3$, be a bounded Lipschitz domain, let $\left(u^{\varepsilon}\right)_{\varepsilon} \subset H^{1}\left(Q ; \mathbb{R}^{m}\right)$ be a sequence of $\mathbb{R}^{m}$-valued functions with

$$
u^{\varepsilon} \rightarrow u \text { weakly in } H^{1}\left(Q, \mathbb{R}^{m}\right) \text { for } \varepsilon \rightarrow 0 .
$$

Let $h>0$ be arbitrary. Then there exists an adapted grid, i.e. $Q_{h} \subset Q$ with a triangulation $\mathbb{T}_{h}$ as in (2.2), such that the following compensated compactness property is satisfied: For every sequence $\left(q^{\varepsilon}\right)_{\varepsilon}$ in $L^{2}\left(Q, \mathbb{R}^{m \times n}\right)$ satisfying

$$
\begin{aligned}
q^{\varepsilon} \rightarrow q & \text { weakly in } L^{2}(Q), \\
f^{\varepsilon}:=\nabla \cdot q^{\varepsilon} \rightarrow f & \text { strongly in } H^{-1}\left(\mathcal{T}_{k} ; \mathbb{R}^{m}\right), \quad \text { for all } \mathcal{T}_{k} \in \mathbb{T}_{h},
\end{aligned}
$$

there holds

$$
\lim _{\varepsilon \rightarrow 0} \int_{Q_{h}} q^{\varepsilon}: \nabla u^{\varepsilon}=\int_{Q_{h}} q: \nabla u .
$$

The regularity of the sequence of grids was not explicitly stated in [20], but the construction shows that a regular sequence can be constructed. Furthermore, the original statement in [20] is for the case $m=1$. However, the result remains valid for every $m \in \mathbb{N}$ (with identical proof).

On the other hand, we must generalize to time dependent functions $u^{\varepsilon} \rightarrow u$ in $L^{2}\left(0, T ; H^{1}(Q)\right)$. To treat a time dependent situation, we must use a time-discretized setting; the subsequent version of Theorem 2.1 allows to treat sequences of functions $u^{\varepsilon} \rightarrow u$ in $H^{1}\left(0, T ; H^{1}(Q)\right)$ due to their additional regularity in time.

Corollary 2.2 (Adapted grids and div-curl property for time-dependent functions). Let $Q \subset \mathbb{R}^{n}, n \leq 3$, be a bounded Lipschitz domain, let $\left(u^{\varepsilon}\right)_{\varepsilon} \subset H^{1}\left(0, T ; H^{1}\left(Q ; \mathbb{R}^{n}\right)\right)$ be a sequence of functions with

$$
u^{\varepsilon} \rightarrow u \text { weakly in } H^{1}\left(0, T ; H^{1}(Q)\right) \text { for } \varepsilon \rightarrow 0 .
$$


Let $h>0$ be arbitrary and let $0 \leq t_{0}<t_{1}<\ldots<t_{K} \leq T$ be points in $[0, T]$. Then there exists a adapted grid $Q_{h} \subset Q$ with a triangulation $\mathbb{T}_{h}$ of $Q_{h}$ as in (2.2) such that the following compensated compactness result holds.

For every family of sequences $\left(q_{k}^{\varepsilon}\right)_{\varepsilon}$ in $L^{2}\left(Q, \mathbb{R}^{n \times n}\right)$, satisfying, for every $k \leq K$,

$$
\begin{aligned}
q_{k}^{\varepsilon} \rightarrow q_{k} & \text { weakly in } L^{2}(Q), \\
f_{k}^{\varepsilon}:=\nabla \cdot q_{k}^{\varepsilon} \rightarrow f_{k} & \text { strongly in } H^{-1}\left(\mathcal{T}_{i} ; \mathbb{R}^{n \times n}\right), \quad \text { for all } \mathcal{T}_{i} \in \mathbb{T}_{h},
\end{aligned}
$$

there holds, for every $k \leq K$, evaluating $\nabla u^{\varepsilon}\left(t_{k}\right)$ in the sense of traces,

$$
\lim _{\varepsilon \rightarrow 0} \int_{Q_{h}} q_{k}^{\varepsilon}: \nabla u^{\varepsilon}\left(t_{k}\right)=\int_{Q_{h}} q_{k}: \nabla u\left(t_{k}\right)
$$

Proof. The function space $H^{1}\left(0, T ; H^{1}(Q)\right)$ embeds continuously into $C\left([0, T] ; H^{1}(Q)\right)$, hence $u^{\varepsilon}\left(t_{k}\right) \rightarrow u\left(t_{k}\right)$ in $H^{1}(Q)$ for every $k \in\{0,1, \ldots, K\}$. This allows to consider $\left(u^{\varepsilon}\left(t_{0}\right), u^{\varepsilon}\left(t_{1}\right), \ldots, u^{\varepsilon}\left(t_{K}\right)\right)$ as a sequence of functions in $H^{1}\left(Q ; \mathbb{R}^{(K+1) n}\right)$ and to apply Theorem 2.1.

\subsection{Discretization, projections, and a new function space}

In this section, we assume that a sequence $h \rightarrow 0$, a sequence of polygonal domains $Q_{h} \subset Q$, and a sequence of triangulations $\mathbb{T}_{h}$ as described in (2.2) are given. We consider the corresponding finite element space of continuous and piecewise affine functions with vanishing boundary values,

$$
Y_{h}:=\left\{\phi \in H_{0}^{1}(Q)|\phi|_{\mathcal{T}_{k}} \text { is affine } \forall \mathcal{T}_{k} \in \mathbb{T}_{h}, \phi \equiv 0 \text { on } Q \backslash Q_{h}\right\}
$$

Boundary conditions. We start by transforming the boundary data $w$ into a piecewise affine function. To this end, we extend the triangulation of $Q_{h}$ by a finite number of simplices with diameter not greater than $h$ to obtain a grid $\tilde{\mathbb{T}}_{h}$ that covers $Q$ in the sense that $Q \subset \bigcup_{\mathcal{T}_{k} \in \tilde{\mathbb{T}}_{h}} \mathcal{T}_{k}$. The extended grid $\tilde{\mathbb{T}}_{h}$ allows to introduce the space $\tilde{Y}_{h}:=\left\{\phi \in H^{1}(Q)|\phi|_{\mathcal{T}_{k} \cap Q}\right.$ is affine for every $\left.\mathcal{T}_{k} \in \tilde{\mathbb{T}}_{h}\right\}$. Denoting by $R_{Q, h}$ the $H^{1}(Q)$ orthogonal projection $H^{1}(Q) \rightarrow \tilde{Y}_{h}$ (the Riesz-projection), we define piecewise affine boundary data as $w_{h}:=R_{Q, h}(w)$.

We note that there holds the strong convergence $w_{h} \rightarrow w$ in $H^{1}\left(0, T ; H^{1}(Q)\right)$ as $h \rightarrow 0$.

Transformation of the right hand side $f$. For a given right hand side $f \in$ $H^{1}\left(0, T ; L^{2}(Q)\right)$ and boundary data $w_{h} \rightarrow w$ in $H^{1}\left(0, T ; H^{1}(Q)\right)$, we consider the following discretized Poisson problem (with $t$ as a parameter):

$$
\text { Find } \quad v_{h}(t) \in w_{h}(t)+Y_{h} \text { with } \int_{Q} \nabla v_{h}(t) \cdot \nabla \phi=\int_{Q} f(t) \phi \quad \forall \phi \in Y_{h} \text {. }
$$

Existence and uniqueness of solutions $v_{h}(t)$ and a uniform a priori estimate for $v_{h} \in$ $H^{1}\left(0, T ; H^{1}(Q)\right)$ can easily be concluded with the Lax-Milgram theorem. 
We use $v_{h}$ to transform the right hand side $f$ into jump conditions across edges of the grid $\mathbb{T}_{h}$. We denote the subset composed by interior interfaces as

$$
\Gamma^{h}:=\left(\bigcup_{k \in \Lambda_{h}} \partial \mathcal{T}_{k}\right) \backslash \partial Q_{h}=\bigcup_{k<j} \Gamma_{k, j}, \quad \Gamma_{k, j}:=\overline{\mathcal{T}}_{k} \cap \overline{\mathcal{T}}_{j} .
$$

We write $\nu_{(k)}$ for the outer normal to $\mathcal{T}_{k}$ on $\partial \mathcal{T}_{k}$. For every function $\varphi: Q \rightarrow \mathbb{R}^{n}$ with the property that the traces of the normal component $\left.\varphi\right|_{\partial \mathcal{T}_{k}} \cdot \nu_{(k)}$ are well defined for every $k \in \Lambda_{h}$, we define the normal jump across $\Gamma_{k, j}$ through

$$
[[\varphi]]_{k j}:=\left.\varphi\right|_{\mathcal{T}_{k}} \cdot \nu_{(k)}+\left.\varphi\right|_{\mathcal{T}_{j}} \cdot \nu_{(j)}=\left(\left.\varphi\right|_{\mathcal{T}_{k}}-\left.\varphi\right|_{\mathcal{T}_{j}}\right) \cdot \nu_{(k)}
$$

The definition guarantees $[[\varphi]]_{k j}=[[\varphi]]_{j k}$. The collection of jumps can be treated as a function on the interfaces, $[[\varphi]]: \Gamma_{h} \rightarrow \mathbb{R}$.

For a given $f$ and with $v_{h}$ from (2.10), we define $g_{h}: \Gamma_{h} \rightarrow \mathbb{R}$ as the function

$$
\left.g_{h}\right|_{\Gamma_{k, j}}:=\left[\left[\nabla v_{h}\right]\right]_{k j} .
$$

We note that, by construction of $v_{h}$, the function $g_{h}: \Gamma_{h} \rightarrow \mathbb{R}$ is constant on each interface $\Gamma_{k, j}$. With this construction, we have transformed the right hand side $f$ into the jump condition $g_{h}$. Indeed, using after each other (2.10), the fact that $\nabla v_{h}$ is constant in each $\mathcal{T}_{k}$, the definition of jumps and (2.11), we find for arbitrary $\phi \in Y_{h}$

$$
\int_{Q} f \phi=\int_{Q} \nabla v_{h} \cdot \nabla \phi=\sum_{k} \int_{\partial \mathcal{T}_{k}}\left(\nabla v_{h} \cdot \nu_{(k)}\right) \phi=\sum_{k<j} \int_{\Gamma_{k, j}}\left[\left[\nabla v_{h}\right]\right]_{k j} \phi=\int_{\Gamma_{h}} g_{h} \phi .
$$

In this sense, we have replaced $f \in L^{2}(Q)$ by the measure $\left.g_{h} \mathcal{H}^{n-1}\right|_{\Gamma_{h}}$.

Remark 6. In every time instance $t$, the finite element solution $v_{h}=v_{h}(t)$ of $(2.10)$ solves, by construction of $g_{h}$, the problem

$$
v_{h} \in w_{h}+Y_{h} \quad \text { with } \quad\left[\left[\nabla v_{h}\right]\right]_{k j}=\left.g_{h}\right|_{\Gamma_{k, j}} \forall k<j .
$$

Vice versa, every solution to problem (2.13) is a solution to problem (2.10). This follows from the fact that, in a connected set $Q$, the normal jumps of the gradient across interfaces determine a piecewise affine function uniquely.

Definition 2.3 (Needle problem function space). Let $Q \subset \mathbb{R}^{n}$ be a Lipschitz domain with a triangulation $\mathbb{T}_{h}$ of $Q_{h} \subset Q$ with interior interfaces $\Gamma_{h}$. We define the needle problem function space

$$
\mathcal{N}_{h}:=\left\{\phi \in H_{0}^{1}(Q)|\phi|_{\partial \mathcal{T}_{k}} \text { is affine for all } \mathcal{T}_{k} \in \mathbb{T}_{h}, \phi \equiv 0 \text { on } Q \backslash Q_{h}\right\} .
$$

We furthermore introduce projections $\mathcal{F}_{h}: \mathcal{N}_{h} \rightarrow Y_{h} \subset \mathcal{N}_{h}$ as follows: a function $u \in \mathcal{N}_{h}$ (which is piecewise affine on edges) is mapped to the piecewise affine extension of the values of $u$ on edges. More precisely, $\mathcal{F}_{h}(u): Q \rightarrow \mathbb{R}$ is the function

$$
\mathcal{F}_{h}(u) \in Y_{h},\left.\quad \mathcal{F}_{h}(u)\right|_{\Gamma_{h}}=\left.u\right|_{\Gamma_{h}} .
$$

We use the construction also in affine spaces: For a boundary condition given by $w \in H^{1}\left(0, T ; H^{1}(\Omega)\right)$ with a piecewise affine discretization $w_{h}$, we define $\mathcal{F}_{h}^{w}$ : $w_{h}+\mathcal{N}_{h} \rightarrow w_{h}+Y_{h}$ by $\mathcal{F}_{h}^{w}(u):=w_{h}+\mathcal{F}_{h}\left(u-w_{h}\right)$. 
We have the following quite elementary properties of $\mathcal{F}_{h}$ (see Lemma 2.5 in [20]).

Lemma 2.4. Let $\mathcal{F}_{h}: \mathcal{N}_{h} \rightarrow Y_{h} \subset \mathcal{N}_{h}$ be the projections of Definition 2.3. These projections have the following properties.

(i) $\nabla \mathcal{F}_{h}(u)(x)=f_{\mathcal{T}_{k}} \nabla u$ for $x \in \mathcal{T}_{k}$.

(ii) Let $h>0$ be fixed and let $u^{\varepsilon} \in \mathcal{N}_{h}$ be weakly convergent, $u^{\varepsilon} \rightarrow u$ in $H^{1}(Q)$ as $\varepsilon \rightarrow 0$. Then

$$
\mathcal{F}_{h}\left(u^{\varepsilon}\right) \underset{\varepsilon}{\rightarrow} \mathcal{F}_{h}(u) \text { weakly in } H^{1}(Q) \text {. }
$$

(iii) Let $u_{h} \in \mathcal{N}_{h}$ be weakly convergent, $u_{h} \rightarrow u$ in $H^{1}(Q)$ as $h \rightarrow 0$. Then

$$
\mathcal{F}_{h}\left(u_{h}\right) \underset{h}{\rightarrow} u \quad \text { weakly in } H^{1}(Q) \text {. }
$$

Properties (i) and (ii) hold also for the affine counterparts $\mathcal{F}_{h}^{w}$, property (iii) for $\mathcal{F}_{h}^{w_{h}}$ along any sequence $w_{h} \rightarrow w$ in $H^{1}(Q)$.

The projection $\mathcal{F}_{h}$ allows to formulate a variant of (2.12) that holds for every function $\phi \in \mathcal{N}_{h}$ in the needle problem function space (i.e. affine on edges):

$$
\int_{Q} f \cdot \mathcal{F}_{h}(\phi)=\int_{\Gamma_{h}} g_{h} \mathcal{F}_{h}(\phi)=\int_{\Gamma_{h}} g_{h} \phi .
$$

The $L^{2}$-projection. For a Lipschitz domain $Q \subset \mathbb{R}^{n}$ with triangulation $\mathbb{T}_{h}$ of $Q_{h} \subset$ $Q$ and a piecewise affine function $w_{h}$ prescribing a boundary condition, we denote the $L^{2}(Q)$-orthogonal projection onto the finite element space $Y_{h}$ by $\mathcal{P}_{h}: L^{2}(Q) \rightarrow Y_{h}$. We denote the affine projection by $\mathcal{P}_{h}^{w}(u):=w_{h}+\mathcal{P}_{h}\left(u-w_{h}\right)$. The following Lemma collects some properties of $\mathcal{P}_{h}$ and $\mathcal{P}_{h}^{w}$, which are standard in the theory of finite element methods.

Lemma 2.5. For regular sequences of grids, the $L^{2}$-orthogonal projections $\mathcal{P}_{h}: L^{2}(Q) \rightarrow$ $Y_{h}$ have the following properties:

(i) With a constant $C$ independent of h holds $\left\|\mathcal{P}_{h}(u)\right\|_{H^{1}(Q)} \leq C\|u\|_{H^{1}(Q)}$.

(ii) For $u^{\varepsilon} \rightarrow u$ in $H^{1}(Q)$ weakly holds $\mathcal{P}_{h}\left(u^{\varepsilon}\right) \rightarrow \mathcal{P}_{h}(u)$ strongly in $H^{1}(Q)$.

(iii) For $u \in H_{0}^{1}(Q) \cap H^{2}(Q)$ holds inf $\left\{\|\chi-u\|_{H^{1}(Q)}: \chi \in Y_{h}\right\} \leq C h\|u\|_{H^{2}(Q)}$.

(iv) For $u \in H_{0}^{1}(Q)$, projections converge strongly, $\mathcal{P}_{h}(u) \rightarrow u$ in $H^{1}(Q)$ as $h \rightarrow 0$.

Proof. For the first and third statement we refer to [4], Corollary 7.8, Lemma 7.9 and Proposition 6.8. The second statement is an immediate consequence of the continuity of $\mathcal{P}_{h}$ and the fact that $Y_{h}$ has a finite dimension.

The fourth statement is used less, but it is an elementary consequence of the other properties. Let $u \in H_{0}^{1}(Q)$ be fixed. Exploiting density, we can approximate with $u^{\delta} \in H_{0}^{1}(Q) \cap H^{2}(Q)$ and calculate with the triangle inequality and (i)

$$
\begin{aligned}
\left\|u-\mathcal{P}_{h}(u)\right\|_{H_{0}^{1}(Q)} & \leq\left\|u-u^{\delta}\right\|_{H_{0}^{1}(Q)}+\left\|u^{\delta}-\mathcal{P}_{h}\left(u^{\delta}\right)\right\|_{H_{0}^{1}(Q)}+\left\|\mathcal{P}_{h}\left(u^{\delta}\right)-\mathcal{P}_{h}(u)\right\|_{H_{0}^{1}(Q)} \\
& \stackrel{(i)}{\leq}(1+C)\left\|u^{\delta}-u\right\|_{H_{0}^{1}(Q)}+\left\|\mathcal{P}_{h}\left(u^{\delta}\right)-u^{\delta}\right\|_{H_{0}^{1}(Q)} .
\end{aligned}
$$


We now apply (iii) to the function $u^{\delta}$. For every $h>0$ we find a function $u_{h}$ with $\left\|u^{\delta}-u_{h}\right\|_{H^{1}} \leq C h\left\|u^{\delta}\right\|_{H^{2}}$. Inserting above, we obtain with the triangle inequality

$$
\begin{aligned}
\left\|u-\mathcal{P}_{h}(u)\right\|_{H_{0}^{1}(Q)} & \leq(1+C)\left\|u^{\delta}-u\right\|_{H_{0}^{1}(Q)}+\left\|\mathcal{P}_{h}\left(u^{\delta}\right)-u_{h}\right\|_{H_{0}^{1}(Q)}+\left\|u_{h}-u^{\delta}\right\|_{H_{0}^{1}(Q)} \\
& \stackrel{(i)}{\leq}(1+C)\left(\left\|u^{\delta}-u\right\|_{H_{0}^{1}(Q)}+\left\|u_{h}-u^{\delta}\right\|_{H_{0}^{1}(Q)}\right),
\end{aligned}
$$

where we exploited $\mathcal{P}_{h}\left(u_{h}\right)=u_{h}$ in the last step. Choosing first $u^{\delta}$ sufficiently close to $u$ and then $h>0$ small, we conclude smallness of the expression on the right hand side.

\subsection{The needle problem of plasticity}

We are now in the position to formulate the needle problem of plasticity. Essentially, we demand that the full set of equations is satisfied - with the only exception that we do not impose $-\nabla \cdot \sigma=f$ on interior grid edges. This reduction in the set of equations reflects the fact that we impose a side-condition on solutions: we demand that $u$ is in the needle space, i.e. that $u$ is affine on interior grid edges.

Definition 2.6 (The needle problem of plasticity). The needle problem is to find $u_{h}^{\varepsilon} \in w_{h}+H^{1}\left(0, T ; \mathcal{N}_{h}\right), e_{h}^{\varepsilon}, p_{h}^{\varepsilon}, \sigma_{h}^{\varepsilon} \in H^{1}\left(0, T ; L^{2}\left(Q_{h} ; \mathbb{R}_{s}^{n \times n}\right)\right)$, such that $p_{h}^{\varepsilon}(0)=0$ and

$$
\int_{0}^{T} \int_{Q_{h}} \sigma_{h}^{\varepsilon}: \nabla \varphi=\int_{0}^{T} \int_{\Gamma_{h}} g_{h} \cdot \varphi \quad \forall \varphi \in L^{2}\left(0, T ; \mathcal{N}_{h}\right),
$$

and almost everywhere in $Q_{h}$ holds

$$
\begin{aligned}
\nabla^{s} u_{h}^{\varepsilon} & =e_{h}^{\varepsilon}+p_{h}^{\varepsilon}, \quad C_{\varepsilon} \sigma_{h}^{\varepsilon}=e_{h}^{\varepsilon}, \\
\partial_{t} p_{h}^{\varepsilon} & \in \partial \Psi_{\varepsilon}\left(\sigma_{h}^{\varepsilon}-B_{\varepsilon} p_{h}^{\varepsilon}\right) .
\end{aligned}
$$

We set $\sigma_{h}^{\varepsilon}=p_{h}^{\varepsilon}=e_{h}^{\varepsilon}=0$ on $Q \backslash Q_{h}$ and $u_{h}^{\varepsilon}=w_{h}$ on $Q \backslash Q_{h}$. We note that, by (2.15), relation $(2.16)$ can also be written as

$$
\int_{0}^{T} \int_{Q_{h}} \sigma_{h}^{\varepsilon}: \nabla \varphi=\int_{0}^{T} \int_{Q_{h}} f \cdot \mathcal{F}_{h}(\varphi) \quad \forall \varphi \in L^{2}\left(0, T ; \mathcal{N}_{h}\right) .
$$

Theorem 2.7 (Existence and uniqueness for the needle problem). Let $Q \subset \mathbb{R}^{n}$ be open and bounded and let the data satisfy (1.2)-(1.5) as in Theorem 1.6. Then, for every $\varepsilon>0$ and every $h>0$, there exists a unique solution to the needle problem of Definition 2.6. The sequence of solutions satisfies the uniform estimate

$$
\left\|u_{h}^{\varepsilon}\right\|_{\mathcal{V}_{1}^{1}}+\left\|e_{h}^{\varepsilon}\right\|_{\mathcal{V}_{0}^{1}}+\left\|p_{h}^{\varepsilon}\right\|_{\mathcal{V}_{0}^{1}}+\left\|\sigma_{h}^{\varepsilon}\right\|_{\mathcal{V}_{0}^{1}} \leq C(w, f)
$$

We recall that $\mathcal{V}_{1}^{1}=H^{1}\left(0, T ; H_{0}^{1}(Q)\right)$ and $\mathcal{V}_{0}^{1}=H^{1}\left(0, T ; L^{2}\left(Q ; \mathbb{R}_{s}^{n \times n}\right)\right)$.

The existence proof for the needle problem is analogous to the existence proof for the original problem, see Theorem 1.2: A Galerkin scheme is constructed, uniform estimates for the corresponding discrete problems allow to select a weakly convergent subsequence, the solution properties are checked for the limit. We nevertheless sketch the proof of Theorem 2.7 in the appendix. 


\section{The effective system and properties of $\Sigma$}

In this section, we collect some properties of the operator $\Sigma$. Since $\Sigma$ is defined through the averaging property of Definition 1.3, all properties of $\Sigma$ must be derived from Definition 1.3. We start with some basic facts that will allow to derive a uniqueness result in Section 3.2.

\subsection{An estimate for $\Sigma$}

Regarding initial values, we have already noted in Remark 3 after Definition 1.3 that, for $t=0$, an elasticity system is averaged. For any $\xi \in H^{1}\left(0, T ; \mathbb{R}_{s}^{n \times n}\right)$, there holds

$$
\Sigma(\xi)(0)=\lim _{\varepsilon \rightarrow 0} f_{\mathcal{T}} \sigma^{\varepsilon}(0)=\lim _{\varepsilon \rightarrow 0} f_{\mathcal{T}} C_{\varepsilon}^{-1} \nabla^{s} u^{\varepsilon}(0)=C_{*}^{-1} \xi(0) .
$$

Lemma 3.1. Let $C_{\varepsilon}, B_{\varepsilon}$ and $\Psi_{\varepsilon}$ satisfy (1.2)-(1.5) and allow averaging with the causal operators $\Sigma$ and $\Pi$. Then there is a constant $C>0$ such that, for two inputs $\xi_{1}, \xi_{2} \in H^{1}\left(0, T ; \mathbb{R}_{s}^{n \times n}\right)$ there holds, for almost every $t \in[0, T]$,

$$
\begin{aligned}
& \left|\xi_{1}-\xi_{2}\right|^{2}(t)+\left(\Sigma\left(\xi_{1}\right)-\Sigma\left(\xi_{2}\right)\right)^{2}(t)+\left(\Pi\left(\xi_{1}\right)-\Pi\left(\xi_{2}\right)\right)^{2}(t) \\
& \leq C\left(\left|\xi_{1}-\xi_{2}\right|^{2}(0)+\int_{0}^{t}\left(\Sigma\left(\xi_{1}\right)-\Sigma\left(\xi_{2}\right)\right): \partial_{t}\left(\xi_{1}-\xi_{2}\right)\right) .
\end{aligned}
$$

Proof. The lemma is a consequence of the energy estimate for the plasticity system. Given two inputs $\xi_{1}, \xi_{2} \in H^{1}\left(0, T ; \mathbb{R}_{s}^{n \times n}\right)$, we exploit the definition of $\Sigma\left(\xi_{i}\right)$ : For $\varepsilon>0$ and $i \in\{1,2\}$ we consider the $\varepsilon$-plasticity problem on a simplex $\mathcal{T} \subset \mathbb{R}^{n}$, i.e. we consider solutions $u_{i}^{\varepsilon}(t, x)=\xi_{i}(t) \cdot x+v_{i}^{\varepsilon}(t, x)$ with $v_{i}^{\varepsilon} \in H^{1}\left(0, T ; H_{0}^{1}(\mathcal{T})\right)$ of

$$
\begin{gathered}
\int_{0}^{T} \int_{\mathcal{T}} \sigma_{i}^{\varepsilon}: \nabla^{s} \varphi=0 \quad \forall \varphi \in L^{2}\left(0, T ; H_{0}^{1}(\mathcal{T})\right), \\
\partial_{t} p_{i}^{\varepsilon} \in \partial \Psi_{\varepsilon}\left(\sigma_{i}^{\varepsilon}-B_{\varepsilon} p_{i}^{\varepsilon}\right), \quad \nabla^{s} u_{i}^{\varepsilon}=C_{\varepsilon} \sigma_{i}^{\varepsilon}+p_{i}^{\varepsilon},
\end{gathered}
$$

with initial condition $p_{i}^{\varepsilon}(0)=0$.

The initial values (in the sense of traces) satisfy $\nabla \cdot\left(\sigma_{1}^{\varepsilon}(0)-\sigma_{2}^{\varepsilon}(0)\right)=0$. Testing this relation with the admissible test functions $u_{1}^{\varepsilon}(., 0)-\xi_{1}(0) \cdot x$ and $u_{2}^{\varepsilon}(., 0)-\xi_{2}(0) \cdot x$ yields, using $\sigma_{i}^{\varepsilon}(0)=C_{\varepsilon}^{-1} \nabla^{s} u_{i}^{\varepsilon}(0)$,

$$
0=\int_{\mathcal{T}} C_{\varepsilon}^{-1} \nabla\left(u_{1}^{\varepsilon}-u_{2}^{\varepsilon}\right)(0):\left(\nabla^{s} u_{1}^{\varepsilon}-\xi_{1}-\nabla^{s} u_{2}^{\varepsilon}+\xi_{2}\right)(0) .
$$

This yields

$$
\left\|\left(\nabla u_{1}^{\varepsilon}-\nabla u_{2}^{\varepsilon}\right)(0)\right\|_{L^{2}(\mathcal{T})}^{2}+\left\|\left(\sigma_{1}^{\varepsilon}-\sigma_{2}^{\varepsilon}\right)(0)\right\|_{L^{2}(\mathcal{T})}^{2} \leq C\left|\xi_{1}-\xi_{2}\right|^{2}(0) .
$$

For positive time instances $t \in(0, T)$ we can now evaluate the expression from the right hand side of (3.2). In equation $(i)$ below, we use $\varphi=\partial_{t}\left(u_{1}^{\varepsilon}-u_{2}^{\varepsilon}-\xi_{1} \cdot x+\xi_{2} \cdot x\right)$ as a test function in (3.3). In inequality (ii) below, we exploit the flow rule, inserting $B_{\varepsilon} p_{1}^{\varepsilon}-B_{\varepsilon} p_{2}^{\varepsilon}$; we use the monotonicity of the subdifferential, which reads $\left(A_{1}-A_{2}\right)$. $\left(\partial \Psi_{\varepsilon}\left(A_{1}\right)-\partial \Psi_{\varepsilon}\left(A_{2}\right)\right) \geq 0$

$$
\begin{gathered}
\int_{0}^{t}\left(\Sigma\left(\xi_{1}\right)-\Sigma\left(\xi_{2}\right)\right): \partial_{t}\left(\xi_{1}-\xi_{2}\right)=\lim _{\varepsilon \rightarrow 0} \int_{0}^{t} f_{\mathcal{T}}\left(\sigma_{1}^{\varepsilon}-\sigma_{2}^{\varepsilon}\right): \partial_{t}\left(\xi_{1}-\xi_{2}\right) \\
\stackrel{(i)}{=} \lim _{\varepsilon \rightarrow 0} \int_{0}^{t} f_{\mathcal{T}}\left(\sigma_{1}^{\varepsilon}-\sigma_{2}^{\varepsilon}\right): \partial_{t} \nabla^{s}\left(u_{1}^{\varepsilon}-u_{2}^{\varepsilon}\right)
\end{gathered}
$$




$$
\begin{aligned}
& =\lim _{\varepsilon \rightarrow 0} \int_{0}^{t} f_{\mathcal{T}}\left(\sigma_{1}^{\varepsilon}-\sigma_{2}^{\varepsilon}\right): \partial_{t}\left(C_{\varepsilon}\left(\sigma_{1}^{\varepsilon}-\sigma_{2}^{\varepsilon}\right)+\left(p_{1}^{\varepsilon}-p_{2}^{\varepsilon}\right)\right) \\
& \stackrel{(i i)}{\geq} \limsup _{\varepsilon \rightarrow 0} \int_{0}^{t} f_{\mathcal{T}} \frac{1}{2} \frac{d}{d t}\left(\left|\sigma_{1}^{\varepsilon}-\sigma_{2}^{\varepsilon}\right|_{C_{\varepsilon}}^{2}+\left|p_{1}^{\varepsilon}-p_{2}^{\varepsilon}\right|_{B_{\varepsilon}}^{2}\right) \\
& =\left.\frac{1}{2} \limsup _{\varepsilon \rightarrow 0} f_{\mathcal{T}}\left(\left|\sigma_{1}^{\varepsilon}-\sigma_{2}^{\varepsilon}\right|_{C_{\varepsilon}}^{2}+\left|p_{1}^{\varepsilon}-p_{2}^{\varepsilon}\right|_{B_{\varepsilon}}^{2}\right)\right|_{0} ^{t} .
\end{aligned}
$$

The averaging property implies the inequality

$$
\left(\Sigma\left(\xi_{1}\right)-\Sigma\left(\xi_{2}\right)\right)^{2}(t) \leq \limsup _{\varepsilon \rightarrow 0} f_{\mathcal{T}}\left|\sigma_{1}^{\varepsilon}-\sigma_{2}^{\varepsilon}\right|^{2}(t),
$$

and the analogous inequality for $\Pi$. Together with (3.5) and (3.6), this yields (3.2) for the $\Sigma$ - and $\Pi$-differences. The estimate for $\xi_{1}(t)-\xi_{2}(t)$ follows from the fact that $\xi_{i}(t)$ is the average of $\nabla^{s} u_{i}^{\varepsilon}$, which is controlled by $\sigma_{i}^{\varepsilon}$ and $p_{i}^{\varepsilon}$.

\subsection{Uniqueness results for the effective problem}

We recall that uniqueness is a standard property of plasticity equations with hardening, compare also our Theorems 1.2 and 2.7. However, the operator $\Sigma$ is not a standard plasticity operator, but it is given only implicitely through the averaging property. With the help of Lemma 3.1, we can nevertheless derive uniqueness results. We start with the discretized problem.

Lemma 3.2 (Uniqueness for the discretized effective problem). Let the data be as in (1.2)-(1.5), allowing averaging with the causal operator $\Sigma$. Let $\mathbb{T}_{h}:=\left\{\mathcal{T}_{k}\right\}_{k \in \Lambda_{h}}$ be a grid for $Q$, let $w_{h} \in H^{1}\left(0, T ; H^{1}(Q)\right)$ be piecewise affine, prescribing boundary data, let $f \in H^{1}\left(0, T ; L^{2}(Q)\right)$ be a right hand side. We consider the following discretized effective problem for $u_{h} \in w_{h}+H^{1}\left(0, T ; Y_{h}\right)$ :

$$
\int_{0}^{T} \int_{Q} \Sigma\left(\nabla^{s} u_{h}\right): \nabla \varphi=\int_{0}^{T} \int_{Q} f \cdot \varphi \quad \forall \varphi \in L^{2}\left(0, T ; Y_{h}\right) .
$$

There exists at most one solution $u_{h}$ to (3.7).

Proof. Let $u_{h, 1}, u_{h, 2}$ be two solutions to (3.7). As solutions of a discretized elasticity problem with boundary data $w_{h}(0)$ and right hand side $f(0)$, the initial values coincide, $u_{h, 1}(0)=u_{h, 2}(0)$.

By definition of $Y_{h}$ in (2.9), for each simplex $\mathcal{T}_{k}$ of the discretization, we find matrices $\xi_{1, k}, \xi_{2, k} \in H^{1}\left(0, T ; \mathbb{R}_{s}^{n \times n}\right)$ and vectors $a_{1, k}, a_{2, k} \in H^{1}\left(0, T ; \mathbb{R}^{n}\right)$, such that

$$
\left.u_{h, i}\right|_{\mathcal{T}_{k}}(t, x)=\xi_{i, k}(t) \cdot x+a_{i, k}(t) .
$$

Since the deformations coincide for $t=0$, we have $\xi_{1, k}(0)=\xi_{2, k}(0)$ and $a_{1, k}(0)=a_{2, k}(0)$ for all $k$. We use $\varphi=\partial_{t}\left(u_{h, 1}-u_{h, 2}\right) \chi_{[0, t]}$ as a test function in (3.7) (for $i=1$ and $i=2$ ) and obtain

$$
\begin{aligned}
0 & \stackrel{(3.7)}{=} \int_{0}^{t} \int_{Q}\left(\Sigma\left(\nabla^{s} u_{h, 1}\right)-\Sigma\left(\nabla^{s} u_{h, 2}\right)\right): \partial_{t}\left(\nabla^{s} u_{h, 1}-\nabla^{s} u_{h, 2}\right) \\
& =\sum_{k} \int_{0}^{t} \int_{\mathcal{T}_{k}}\left(\Sigma\left(\xi_{1, k}\right)-\Sigma\left(\xi_{2, k}\right)\right): \partial_{t}\left(\xi_{1, k}-\xi_{2, k}\right) .
\end{aligned}
$$


Together with $\xi_{1, k}(0)=\xi_{2, k}(0)$, inequality (3.2) provides $\xi_{1, k}(t)=\xi_{2, k}(t)$ for a.e. $t \in$ $[0, T]$, and hence also $u_{h, 1}(t)=u_{h, 2}(t)$ for a.e. $t \in[0, T]$.

Proposition 3.3 (Uniqueness for the effective problem). Let the causal operator $\Sigma$ be the averaged operator for data as in (1.2)-(1.5). Let $w \in H^{1}\left(0, T ; H^{1}(Q)\right)$ prescribe boundary data and let $f \in H^{1}\left(0, T ; L^{2}(Q)\right)$ be a right hand side. Then there is at most one solution $u \in w+H^{1}\left(0, T ; H_{0}^{1}(Q)\right)$ to the effective problem (1.13), i.e.

$$
\int_{0}^{T} \int_{Q} \Sigma\left(\nabla^{s} u\right): \nabla^{s} \varphi=\int_{0}^{T} \int_{Q} f \cdot \varphi \quad \forall \varphi \in L^{2}\left(0, T ; H_{0}^{1}(Q)\right) .
$$

Proof. Let $u_{1}, u_{2}$ be two solutions of (3.8), we write $\xi_{i}:=\nabla^{s} u_{i}$ for the symmetrized gradients. Once more, we can exploit that the initial values $u_{i}(0)$ solve an elasticity problem, $-\nabla \cdot \Sigma\left(\nabla^{s} u_{i}(0) ; 0\right)=-\nabla \cdot C_{*}^{-1} \nabla^{s} u_{i}(0)=f$, compare (3.1). Due to the identical boundary data $w(0)$, this provides $u_{1}(0)=u_{2}(0)$.

The solution space for gradients $\xi_{i}=\nabla^{s} u_{i}$ is

$$
\begin{aligned}
H^{1}\left(0, T ; L^{2}(Q)\right) & =\left\{u \in L^{2}(Q \times(0, T)) \mid \partial_{t} u \in L^{2}(Q \times(0, T))\right\} \\
& =L^{2}\left(Q ; H^{1}(0, T)\right) .
\end{aligned}
$$

This implies that there exists a set $\tilde{Q} \subset Q$ of full measure such that $\xi_{i, x}():.=\xi_{i}(x,)=$. $\nabla^{s} u_{i}(x,.) \in H^{1}\left(0, T ; \mathbb{R}_{s}^{n \times n}\right)$ holds for all $x \in \tilde{Q}$. We can assume that, additionally, there holds $\xi_{1, x}(0)=\xi_{2, x}(0)$ for every $x \in \tilde{Q}$.

We now use $\varphi \partial_{t}\left(u_{1}-u_{2}\right) \chi_{[0, t]}$ as a test function in (3.8) for $i=1,2$ and find

$$
\begin{aligned}
0 & =\int_{0}^{t} \int_{Q}\left(\Sigma\left(\nabla^{s} u_{1}\right)-\Sigma\left(\nabla^{s} u_{2}\right)\right): \partial_{t}\left(\nabla^{s} u_{1}-\nabla^{s} u_{2}\right) \\
& =\int_{0}^{t} \int_{\tilde{Q}}\left(\Sigma\left(\xi_{1, x}\right)-\Sigma\left(\xi_{2, x}\right)\right): \partial_{t}\left(\xi_{1, x}-\xi_{2, x}\right) d x d s \stackrel{(3.2)}{\geq} \frac{1}{C} \int_{\tilde{Q}}\left|\xi_{1, x}(t)-\xi_{2, x}(t)\right|^{2} d x .
\end{aligned}
$$

This provides $\xi_{1}=\xi_{2}$ almost everywhere and hence the uniqueness.

\subsection{The limit $h \rightarrow 0$ in the discretized effective equation}

In the last section, we have obtained uniqueness results for the effective equation (for both, the limit system and the discretized system). We will now use the same method of proof and obtain a convergence result: Solutions $u_{h}$ to the (sequence of) discrete systems converge to a solution $u$ of the space-continuous system.

Proposition 3.4 (Convergence behavior of discrete solutions). Let the causal operator $\Sigma$ be the averaged operator for data as in (1.2)-(1.5). Let $f \in H^{1}\left(0, T ; L^{2}(Q)\right)$ be a right hand side and let $h \rightarrow 0$ be a sequence of positive numbers. For a corresponding sequence of grids $\mathbb{T}_{h}$ let $w_{h} \in H^{1}\left(0, T ; H^{1}(Q)\right)$ be a sequence of piecewise affine functions that prescribe boundary data, such that $w_{h} \rightarrow w$ in $H^{1}\left(0, T ; H^{1}(Q)\right)$. Let $u_{h} \in H^{1}\left(0, T ; H^{1}(Q)\right)$ be a sequence of solutions to the discrete problems (3.7) with boundary condition $w_{h}$, satisfying the uniform bound

$$
\left\|u_{h}\right\|_{H^{1}\left(0, T ; H^{1}(Q)\right)}+\left\|\Sigma\left(\nabla^{s} u_{h}\right)\right\|_{H^{1}\left(0, T ; L^{2}(Q)\right)} \leq C .
$$


Furthermore, let $u \in H^{1}\left(0, T ; H^{1}(Q)\right)$ be a solution to the effective problem (3.8) with the boundary condition $w$. Then there holds

$$
\nabla^{s} u_{h} \rightarrow \nabla^{s} u \quad \text { and } \Sigma\left(\nabla^{s} u_{h}\right) \rightarrow \Sigma\left(\nabla^{s} u\right) \text { and } \Pi\left(\nabla^{s} u_{h}\right) \rightarrow \Pi\left(\nabla^{s} u\right)
$$

as $h \rightarrow 0$ in the space $L^{\infty}\left(0, T ; L^{2}(Q)\right)$.

Proof. As in the previous proofs, we will use a testing procedure and apply (3.2).

Step 1. Initial values. The initial values $u_{h}(0)$ and $u(0)$ are determined by a discretized and a continuous elasticity problem. Using (3.1), from equations (3.7) and (3.8) we obtain

$$
\int_{Q} C_{*}^{-1} \nabla^{s} u_{h}(0): \nabla^{s} \varphi=\int_{Q} f \cdot \varphi \text { and } \int_{Q} C_{*}^{-1} \nabla^{s} u(0): \nabla^{s} \psi=\int_{Q} f \cdot \psi
$$

for every $\varphi \in Y_{h}$ and $\psi \in H_{0}^{1}\left(Q ; \mathbb{R}^{n}\right)$. We use $\varphi:=\psi:=\left(u_{h}-\mathcal{P}_{h}^{w}(u)\right)(0)$ as a test function in both equations. We subtract and obtain

$$
\int_{Q} C_{*}^{-1} \nabla^{s}\left(u_{h}-u\right)(0): \nabla^{s}\left(u_{h}-\mathcal{P}_{h}^{w}(u)\right)(0)=0 .
$$

We expand the second factor as $u_{h}-\mathcal{P}_{h}^{w}(u)=u_{h}-u+u-\mathcal{P}_{h}^{w}(u)$ and conclude with the Cauchy-Schwarz inequality: The strong convergence $\mathcal{P}_{h}^{w}(u(0)) \rightarrow u(0)$ in $H^{1}(Q)$ of Lemma 2.5, item (iv) (which remains valid for strongly convergent boundary values $w_{h}$ ) provides $u_{h}(0) \rightarrow u(0)$ strongly in $H^{1}(Q)$.

Step 2. Positive times. Also in this step, we use differences of solutions as test functions. In the subsequent calculation, the first equality follows from (3.8) with the test function $\partial_{t}\left(u-u_{h}-w+w_{h}\right) \chi_{[0, t]}$, the second equality follows from (3.7) with the test function $\partial_{t}\left(\mathcal{P}_{h}^{w}(u)-u_{h}\right) \chi_{[0, t]}$. We find

$$
\begin{aligned}
\int_{Q} \int_{0}^{t}(\Sigma & \left.\left(\nabla^{s} u\right)-\Sigma\left(\nabla^{s} u_{h}\right)\right): \partial_{t} \nabla^{s}\left(u-u_{h}\right) \\
\stackrel{(3.8)}{=} & \int_{Q} \int_{0}^{t} f \cdot \partial_{t}\left(u-u_{h}-w+w_{h}\right)+\int_{Q} \int_{0}^{t} \Sigma\left(\nabla^{s} u\right): \partial_{t} \nabla^{s}\left(w-w_{h}\right) \\
& -\int_{Q} \int_{0}^{t} \Sigma\left(\nabla^{s} u_{h}\right): \partial_{t} \nabla^{s}\left(u-\mathcal{P}_{h}^{w}(u)+\mathcal{P}_{h}^{w}(u)-u_{h}\right) \\
& \stackrel{(3.7)}{=}=\int_{Q} \int_{0}^{t} f \cdot \partial_{t}\left(u-u_{h}-w+w_{h}\right)-\int_{Q} \int_{0}^{t} f: \partial_{t}\left(\mathcal{P}_{h}^{w}(u)-u_{h}\right) \\
& -\int_{Q} \int_{0}^{t} \Sigma\left(\nabla^{s} u_{h}\right): \partial_{t} \nabla^{s}\left(u-\mathcal{P}_{h}^{w}(u)\right)+\int_{Q} \int_{0}^{t} \Sigma\left(\nabla^{s} u\right): \partial_{t} \nabla^{s}\left(w-w_{h}\right) \\
= & \int_{Q} \int_{0}^{t} f \cdot \partial_{t}\left(u-\mathcal{P}_{h}^{w}(u)-w+w_{h}\right)+\int_{Q} \int_{0}^{t} \Sigma\left(\nabla^{s} u\right): \partial_{t} \nabla^{s}\left(w-w_{h}\right) \\
& -\int_{Q} \int_{0}^{t} \Sigma\left(\nabla^{s} u_{h}\right): \partial_{t} \nabla^{s}\left(u-\mathcal{P}_{h}^{w}(u)\right) .
\end{aligned}
$$

We study the limit $h \rightarrow 0$. The second integral on the right hand side converges to zero because of $w_{h} \rightarrow w$ in $H^{1}\left(0, T ; H^{1}(Q)\right)$. In the first integral we can integrate 
by parts because of $f \in H^{1}\left(0, T ; L^{2}(Q)\right)$; we exploit $u \in C\left([0, T] ; H^{1}(Q)\right)$ to conclude $\mathcal{P}_{h}^{w}(u) \rightarrow u$ in this space (generalizing (iv) of Lemma 2.5), and obtain that also the first integral vanishes in the limit $h \rightarrow 0$. The third integral is treated similarly with the help of the a priori estimate (3.9):

$$
\begin{aligned}
& \left|\int_{Q} \int_{0}^{t} \Sigma\left(\nabla^{s} u_{h}\right): \partial_{t} \nabla^{s}\left(u-\mathcal{P}_{h}^{w}(u)\right)\right| \\
& \quad \leq\left|\int_{Q} \Sigma\left(\nabla^{s} u_{h}\right): \nabla^{s}\left(u-\mathcal{P}_{h}^{w}(u)\right)\right|_{0}^{t}|+| \int_{Q} \int_{0}^{t} \partial_{t} \Sigma\left(\nabla^{s} u_{h}\right): \nabla^{s}\left(u-\mathcal{P}_{h}^{w}(u)\right) \mid \\
& \quad \leq C\left\|u-\mathcal{P}_{h}^{w}(u)\right\|_{C\left([0, T] ; H^{1}(Q)\right)} \rightarrow 0
\end{aligned}
$$

as $h \rightarrow 0$. Together with the convergence of the initial values we have therefore obtained

$$
\int_{Q}\left|\nabla^{s} u(0)-\nabla^{s} u_{h}(0)\right|^{2}+\int_{Q} \int_{0}^{t}\left(\Sigma\left(\nabla^{s} u\right)-\Sigma\left(\nabla^{s} u_{h}\right)\right): \partial_{t}\left(\nabla^{s} u-\nabla^{s} u_{h}\right) \rightarrow 0 .
$$

We can now apply the estimate (3.2) for $\Sigma$ with the arguments $\xi_{1}(x, t)=\nabla^{s} u(x, t)$ and $\xi_{2}(x, t)=\nabla^{s} u_{h}(x, t)$ in almost every point $x \in Q$. We obtain the strong convergences $\nabla^{s} u_{h} \rightarrow \nabla^{s} u, \Sigma\left(\nabla^{s} u_{h}\right) \rightarrow \Sigma\left(\nabla^{s} u\right)$, and $\Pi\left(\nabla^{s} u_{h}\right) \rightarrow \Pi\left(\nabla^{s} u\right)$ in the space $L^{\infty}\left(0, T ; L^{2}(Q)\right)$ as claimed.

\section{Proof of the main theorem}

We recall the sketch of proof of the main theorem in (2.1). In the last section we have obtained the vertical arrow on the right hand side. Our next aim is to justify the other two arrows.

\subsection{Original problem and needle problem}

In this section, we deal with the vertical arrow on the left hand side: We will show that the solution $u_{h}^{\varepsilon}$ of the needle problem (2.16)-(2.18) is close to the solution $u^{\varepsilon}$ of the original problem (1.8)-(1.9).

We assume here that a sequence $\varepsilon \rightarrow 0$ with a solution sequence $u^{\varepsilon}$ is fixed. For a sequence $h \rightarrow 0$ we furthermore fix a sequence $K=K_{h} \rightarrow \infty$ and a family of time discretizations $0=t_{0}^{h}<t_{1}^{h}<\ldots<t_{K}^{h}=T$ with $\max _{k}\left|t_{k+1}^{h}-t_{k}^{h}\right| \leq h$. According to these data $\left(u^{\varepsilon}, h,\left(t_{k}^{h}\right)_{k}\right)$, we choose a sequence of adapted grids as in Corollary 2.2. Furthermore, we project boundary data $w$ with a sequence of functions $w_{h}$ satisfying $w_{h} \rightarrow w$ as in Section 2.3.

Initial data. We start by an analysis of the initial values. The function $u_{h}^{\varepsilon}(0) \in$ $w_{h}^{\varepsilon}(0)+\mathcal{N}_{h}$ solves the stationary needle-problem

$$
\int_{Q}\left(C_{\varepsilon}^{-1} \nabla^{s} u_{h}^{\varepsilon}(0)\right): \nabla \varphi=\int_{Q} f(0) \cdot \varphi \quad \forall \varphi \in \mathcal{N}_{h}
$$

while $u_{h}^{\varepsilon}(0)$ solves the corresponding uncontrained elasticity problem. For this sequence of elliptic problems with constraints, Proposition 2.6 of [20] yields the following 
convergence property:

$\lim _{h \rightarrow 0} \lim _{\varepsilon \rightarrow 0}\left(\left\|\left(\nabla^{s} u^{\varepsilon}-\nabla^{s} u_{h}^{\varepsilon}\right)(0)\right\|_{L^{2}(Q)}+\left\|\left(\sigma^{\varepsilon}-\sigma_{h}^{\varepsilon}\right)(0)\right\|_{L^{2}(Q)}+\left\|\left(p^{\varepsilon}-p_{h}^{\varepsilon}\right)(0)\right\|_{L^{2}(Q)}\right)=0$.

Proposition 4.1 (Comparison of $u_{h}^{\varepsilon}$ and $u^{\varepsilon}$ ). For $\varepsilon \rightarrow 0$ and $h \rightarrow 0$, let the data $\left(u^{\varepsilon}, h,\left(t_{k}^{h}\right)_{k}\right)$ be as described above, and let $u_{h}^{\varepsilon} \in w_{h}+H^{1}\left(0, T ; \mathcal{N}_{h}\right)$ be the solutions to the needle problem (2.16)-(2.18) on the adapted grids. Then there holds

$$
\lim _{h \rightarrow 0} \limsup _{\varepsilon \rightarrow 0}\left(\left\|\nabla^{s} u^{\varepsilon}-\nabla^{s} u_{h}^{\varepsilon}\right\|_{\mathcal{V}^{0, \infty}}+\left\|\sigma^{\varepsilon}-\sigma_{h}^{\varepsilon}\right\|_{\mathcal{V}^{0, \infty}}+\left\|p^{\varepsilon}-p_{h}^{\varepsilon}\right\|_{\mathcal{V}^{0, \infty}}\right)=0
$$

where $\mathcal{V}^{0, \infty}:=L^{\infty}\left(0, T ; L^{2}\left(Q ; \mathbb{R}^{n \times n}\right)\right)$.

The idea of the proof is to use $\partial_{t}\left(u^{\varepsilon}-u_{h}^{\varepsilon}\right)$ as a test function in the original problem (1.8) and in the needle problem (2.16), and to take the difference. There are two difficulties.

1.) The function $\partial_{t}\left(u^{\varepsilon}-u_{h}^{\varepsilon}\right)$ is not a valid test-function. The first problem is that it does not vanish at the boundary. This can be corrected by subtracting the boundary data $w-w_{h}$. The second problem is that $\partial_{t} u^{\varepsilon}$ is not in the needle space. This fact is circumvented by using the projection $\partial_{t}\left(\mathcal{P}_{h} u^{\varepsilon}-u_{h}^{\varepsilon}\right)$ in the needle problem.

2.) In the energy estimate there appears, on the right hand side, an integral over a product of weakly convergent sequences. This integral must be treated with the compensated compactness property of Corollary 2.2 .

Proof. The sequences $u^{\varepsilon}$ and $u_{h}^{\varepsilon}$ satisfy the a priori estimates (1.10) and (2.20). We can therefore extract a subsequence and find limit functions $u, u_{h} \in H^{1}\left(0, T ; H^{1}(Q)\right)$ and $p, p_{h}, \sigma, \sigma_{h} \in H^{1}\left(0, T ; L^{2}(Q)\right)$, such that, as $\varepsilon \rightarrow 0$,

$$
\begin{aligned}
& u^{\varepsilon} \rightarrow u, \quad u_{h}^{\varepsilon} \rightarrow u_{h} \quad \text { weakly in } H^{1}\left(0, T ; H^{1}(Q)\right), \\
& p^{\varepsilon} \rightarrow p, \quad p_{h}^{\varepsilon} \rightarrow p_{h}, \quad \sigma^{\varepsilon} \rightarrow \sigma, \quad \sigma_{h}^{\varepsilon} \rightarrow \sigma_{h} \quad \text { weakly in } H^{1}\left(0, T ; L^{2}(Q)\right) .
\end{aligned}
$$

Step 1: An estimate of energy type. We consider, for $t_{0} \in(0, T)$,

$$
\begin{aligned}
I_{h}^{\varepsilon}:= & \frac{1}{2} \int_{0}^{t_{0}} \frac{d}{d t} \int_{Q}\left(\left|\sigma^{\varepsilon}-\sigma_{h}^{\varepsilon}\right|_{C_{\varepsilon}}^{2}+\left|p^{\varepsilon}-p_{h}^{\varepsilon}\right|_{B_{\varepsilon}}^{2}\right) \\
\stackrel{(*)}{\leq} & \frac{1}{2} \int_{0}^{t_{0}} \frac{d}{d t} \int_{Q}\left(\left|\sigma^{\varepsilon}-\sigma_{h}^{\varepsilon}\right|_{C_{\varepsilon}}^{2}+\left|p^{\varepsilon}-p_{h}^{\varepsilon}\right|_{B_{\varepsilon}}^{2}\right) \\
& +\int_{0}^{t_{0}} \int_{Q} \partial_{t}\left(p^{\varepsilon}-p_{h}^{\varepsilon}\right):\left(\left(\sigma^{\varepsilon}-B_{\varepsilon} p^{\varepsilon}\right)-\left(\sigma_{h}^{\varepsilon}-B_{\varepsilon} p_{h}^{\varepsilon}\right)\right) \\
= & \int_{0}^{t_{0}} \int_{Q}\left(\sigma^{\varepsilon}-\sigma_{h}^{\varepsilon}\right): \partial_{t}\left(C_{\varepsilon}\left(\sigma^{\varepsilon}-\sigma_{h}^{\varepsilon}\right)+\left(p^{\varepsilon}-p_{h}^{\varepsilon}\right)\right) \\
= & \int_{0}^{t_{0}} \int_{Q} \sigma^{\varepsilon}: \partial_{t} \nabla^{s}\left(u^{\varepsilon}-u_{h}^{\varepsilon}\right)-\int_{0}^{t_{0}} \int_{Q} \sigma_{h}^{\varepsilon}: \partial_{t} \nabla^{s}\left(u^{\varepsilon}-u_{h}^{\varepsilon}\right),
\end{aligned}
$$

where in $(*)$ we used the flow rules

$$
\partial_{t} p^{\varepsilon} \in \partial \Psi_{\varepsilon}\left(\sigma^{\varepsilon}-B_{\varepsilon} p^{\varepsilon}\right) \quad \text { and } \quad \partial_{t} p_{h}^{\varepsilon} \in \partial \Psi_{\varepsilon}\left(\sigma_{h}^{\varepsilon}-B_{\varepsilon} p_{h}^{\varepsilon}\right)
$$

and the monotonicity of $\partial \Psi_{\varepsilon}$. 
The first integral on the right hand side of (4.2) is transformed with the $\varepsilon$-equation (1.8); using $\partial_{t}\left(u^{\varepsilon}-u_{h}^{\varepsilon}-w+w_{h}\right)$ as a test function we find

$$
\begin{aligned}
\int_{0}^{t_{0}} & \int_{Q} \sigma^{\varepsilon}: \partial_{t} \nabla^{s}\left(u^{\varepsilon}-u_{h}^{\varepsilon}\right) \\
& =\int_{0}^{t_{0}} \int_{Q} f \cdot \partial_{t}\left(u^{\varepsilon}-u_{h}^{\varepsilon}-w+w_{h}\right)+\int_{0}^{t_{0}} \int_{Q} \sigma^{\varepsilon}: \partial_{t} \nabla^{s}\left(w-w_{h}\right)
\end{aligned}
$$

The second integral in (4.2) is treated with the help of the needle-problem (2.16), using the test function $\partial_{t}\left(\mathcal{P}_{h}^{w}\left(u^{\varepsilon}\right)-u_{h}^{\varepsilon}\right.$ ) and relation (2.15) (which transforms the force $f$ into a force $\left.g_{h}\right)$,

$$
\begin{aligned}
\int_{0}^{t_{0}} & \int_{Q} \sigma_{h}^{\varepsilon}: \partial_{t} \nabla^{s}\left(u^{\varepsilon}-u_{h}^{\varepsilon}\right) \\
& =\int_{0}^{t_{0}} \int_{Q} f \cdot \partial_{t} \mathcal{F}_{h}\left(\mathcal{P}_{h}^{w}\left(u^{\varepsilon}\right)-u_{h}^{\varepsilon}\right)+\int_{0}^{t_{0}} \int_{Q} \sigma_{h}^{\varepsilon}: \partial_{t} \nabla^{s}\left(u^{\varepsilon}-\mathcal{P}_{h}^{w}\left(u^{\varepsilon}\right)\right) .
\end{aligned}
$$

Inserting into (4.2), we obtain

$$
\begin{aligned}
I_{h}^{\varepsilon} \leq & \int_{0}^{t_{0}} \int_{Q} f \cdot \partial_{t}\left(u^{\varepsilon}-\mathcal{P}_{h}^{w}\left(u^{\varepsilon}\right)-w+w_{h}\right)+\int_{0}^{t_{0}} \int_{Q} f \cdot \partial_{t}\left(\mathcal{F}_{h}^{w}\left(u_{h}^{\varepsilon}\right)-u_{h}^{\varepsilon}\right) \\
& \quad+\int_{0}^{t_{0}} \int_{Q} \sigma^{\varepsilon}: \partial_{t} \nabla^{s}\left(w-w_{h}\right)+\int_{0}^{t_{0}} \int_{Q} \sigma_{h}^{\varepsilon}: \partial_{t} \nabla^{s}\left(\mathcal{P}_{h}^{w}\left(u^{\varepsilon}\right)-u^{\varepsilon}\right) \\
=: & I_{h, 1}^{\varepsilon}+I_{h, 2}^{\varepsilon}+I_{h, 3}^{\varepsilon}+I_{h, 4}^{\varepsilon} .
\end{aligned}
$$

We can now exploit the properties of $\mathcal{F}_{h}^{w}$ and $\mathcal{P}_{h}^{w}$ under weak convergence, see Lemma 2.4, item (ii), and Lemma 2.5, item (ii). They provide for the first integral $I_{h, 1}^{\varepsilon} \rightarrow$ $\int_{0}^{t_{0}} \int_{Q} f \cdot \partial_{t}\left(u-\mathcal{P}_{h}^{w}(u)-w+w_{h}\right)$ as $\varepsilon \rightarrow 0$, and for the second integral $I_{h, 2}^{\varepsilon} \rightarrow \int_{0}^{t_{0}} \int_{Q} f$. $\partial_{t}\left(\mathcal{F}_{h}^{w}\left(u_{h}\right)-u_{h}\right)$ as $\varepsilon \rightarrow 0$. In the limit $h \rightarrow 0$, these two integrals vanish due to the convergence $w_{h} \rightarrow w$, the approximation property of $\mathcal{P}_{h}^{w}$ (item (iv) in Lemma 2.5), and the weak convergence of $u_{h}$ to $u$ together with Lemma 2.4, item (iii).

In the third integral $I_{h, 3}^{\varepsilon}$, we use the weak convergence $\sigma^{\varepsilon} \rightarrow \sigma$ and the convergence $w_{h} \rightarrow w$ to conclude $\lim _{h \rightarrow 0} \lim _{\varepsilon \rightarrow 0} I_{h, 3}^{\varepsilon}=0$. We note that the convergence rate for $I_{h, 1}^{\varepsilon}, I_{h, 2}^{\varepsilon}$, and $I_{h, 3}^{\varepsilon}$ is independent of $t_{0}$.

Step 2: Time discretization and compensated compactness. It remains to treat the fourth integral $I_{h, 4}^{\varepsilon}$ of (4.3). We note that, by (2.20), there is $C_{\sigma}>0$ such that $\left\|\partial_{t} \sigma_{h}^{\varepsilon}\right\|_{L^{2}\left(0, T ; L^{2}(Q)\right)} \leq C_{\sigma}$ for all $h, \varepsilon>0$.

The weak convergence $\sigma_{h}^{\varepsilon} \rightarrow \sigma_{h}$ together with the strong convergence $\mathcal{P}_{h}^{w}\left(u^{\varepsilon}\right) \rightarrow$ $\mathcal{P}_{h}^{w}(u)$ as $\varepsilon \rightarrow 0$ provides (with item (iv) in Lemma 2.5)

$$
\int_{0}^{t_{0}} \int_{Q} \sigma_{h}^{\varepsilon}: \partial_{t} \nabla^{s} \mathcal{P}_{h}^{w}\left(u^{\varepsilon}\right) \underset{\varepsilon \rightarrow 0}{\longrightarrow} \int_{0}^{t_{0}} \int_{Q} \sigma_{h}: \partial_{t} \nabla^{s} \mathcal{P}_{h}^{w}(u) \underset{h \rightarrow 0}{\longrightarrow} \int_{0}^{t_{0}} \int_{Q} \sigma: \partial_{t} \nabla^{s} u .
$$

For the other term, we need compensated compactness, which is only available in finitely many points $t_{k}^{h}$. In order to treat the error term that is induced by the discretization, we define an approximation of $\sigma_{h}^{\varepsilon}$, which is piecewise constant in time,

$$
\bar{\sigma}_{h}^{\varepsilon}(t, \cdot):=\sigma_{h}^{\varepsilon}\left(t_{k}^{h}, \cdot\right) \quad \text { for } t \in\left[t_{k}^{h} ; t_{k+1}^{h}\right), k \leq K-1 .
$$


In the same way, we define $\bar{\sigma}_{h}$ as the discretization of $\sigma$. Due to our choice of $t_{k}^{h}$, the approximation satisfies the uniform error estimate

$$
\sup _{t \in[0, T]}\left\|\bar{\sigma}_{h}^{\varepsilon}(t, \cdot)-\sigma_{h}^{\varepsilon}(t, \cdot)\right\|_{L^{2}(Q)} \leq h^{1 / 2}\left\|\partial_{t} \sigma_{h}^{\varepsilon}\right\|_{L^{2}\left(0, T ; L^{2}(Q)\right)} \leq C_{\sigma} h^{1 / 2} .
$$

The remaining part of $I_{h, 4}^{\varepsilon}$ can now be written, choosing $j \leq K-1$ such that $t_{j}^{h}$ satisfies $t_{j}^{h} \leq t_{0} \leq t_{j}^{h}+h$,

$$
\begin{aligned}
\int_{0}^{t_{0}} \int_{Q} \sigma_{h}^{\varepsilon}: \partial_{t} \nabla^{s} u^{\varepsilon}= & \int_{0}^{t_{j}^{h}} \int_{Q} \bar{\sigma}_{h}^{\varepsilon}: \partial_{t} \nabla^{s} u^{\varepsilon} \\
& +\int_{0}^{t_{0}} \int_{Q}\left(\sigma_{h}^{\varepsilon}-\bar{\sigma}_{h}^{\varepsilon}\right): \partial_{t} \nabla^{s} u^{\varepsilon}+\int_{t_{j}^{h}}^{t_{0}} \int_{Q} \bar{\sigma}_{h}^{\varepsilon}: \partial_{t} \nabla^{s} u^{\varepsilon}
\end{aligned}
$$

The last two integrals vanish in the limit $\lim _{h \rightarrow 0} \lim _{\varepsilon \rightarrow 0}$ due to smallness of $\bar{\sigma}_{h}^{\varepsilon}-\sigma_{h}^{\varepsilon}$ and smallness of $t_{0}-t_{j}^{h}$ (independent of the value of $t_{0}$ ). The first integral is written, evaluating $\nabla u^{\varepsilon}\left(t_{k}^{h}\right)$ in the sense of traces, as

$$
\int_{0}^{t_{j}^{h}} \int_{Q} \bar{\sigma}_{h}^{\varepsilon}: \partial_{t} \nabla^{s} u^{\varepsilon}=\sum_{k=0}^{j-1} \int_{Q} \sigma_{h}^{\varepsilon}\left(t_{k}^{h}\right):\left(\nabla^{s} u^{\varepsilon}\left(t_{k+1}^{h}\right)-\nabla^{s} u^{\varepsilon}\left(t_{k}^{h}\right)\right) .
$$

In this form, we can apply Corollary 2.2 to the spatial integrals. We exploit the weak $L^{2}(Q)$-convergence $\sigma_{h}^{\varepsilon}\left(t_{k}^{h}\right) \rightarrow \sigma_{h}\left(t_{k}^{h}\right)$, the fact that $\nabla \cdot \sigma_{h}^{\varepsilon}\left(t_{k}^{h}\right)=0$ holds in each simplex, and the fact that the grid is adapted. Corollary 2.2 yields, in the limit $\varepsilon \rightarrow 0$

$$
\int_{0}^{t_{j}^{h}} \int_{Q} \bar{\sigma}_{h}^{\varepsilon}: \partial_{t} \nabla^{s} u^{\varepsilon} \rightarrow \sum_{k=0}^{j-1} \int_{Q} \sigma_{h}\left(t_{k}^{h}\right):\left(\nabla^{s} u\left(t_{k+1}^{h}\right)-\nabla^{s} u\left(t_{k}^{h}\right)\right)=\int_{0}^{t_{j}^{h}} \int_{Q} \bar{\sigma}_{h}: \partial_{t} \nabla^{s} u .
$$

We have thus shown

$$
\lim _{h \rightarrow 0} \lim _{\varepsilon \rightarrow 0} I_{h, 4}^{\varepsilon}=\int_{0}^{t_{0}} \int_{Q} \sigma: \partial_{t} \nabla^{s} u-\lim _{h \rightarrow 0} \int_{0}^{t_{j}^{h}} \int_{Q} \bar{\sigma}_{h}: \partial_{t} \nabla^{s} u=0 .
$$

We can conclude from the energy estimate (4.3) and the convergence of the initial data that, for almost every $t_{0} \in(0, T)$,

$$
\begin{aligned}
& \lim _{h \rightarrow 0} \limsup _{\varepsilon \rightarrow 0} \int_{Q}\left(\left|\sigma^{\varepsilon}-\sigma_{h}^{\varepsilon}\right|_{C_{\varepsilon}}^{2}+\left|p^{\varepsilon}-p_{h}^{\varepsilon}\right|_{B_{\varepsilon}}^{2}\right)\left(t_{0}\right) \\
& \quad \leq \lim _{h \rightarrow 0} \limsup _{\varepsilon \rightarrow 0} 2 I_{h}^{\varepsilon}+\lim _{h \rightarrow 0} \limsup _{\varepsilon \rightarrow 0} \int_{Q}\left(\left|\sigma^{\varepsilon}-\sigma_{h}^{\varepsilon}\right|_{C_{\varepsilon}}^{2}+\left|p^{\varepsilon}-p_{h}^{\varepsilon}\right|_{B_{\varepsilon}}^{2}\right)(0)=0,
\end{aligned}
$$

Since the convergence rate in all error terms is independent of $t_{0}$, we have verified the claim. The difference $\nabla^{s} u^{\varepsilon}-\nabla^{s} u_{h}^{\varepsilon}$ is controlled by the corresponding differences in stresses $\sigma$ and plastic strains $p$.

\subsection{Stabilization result}

We start our analysis with a variant of the averaging property: The data are allowed to vary with $\varepsilon$. 
Lemma 4.2. Let the coefficients $C_{\varepsilon}, B_{\varepsilon}$ and $\Psi_{\varepsilon}$ allow averaging in the sense of Definition 1.3. For a simplex $\mathcal{T} \subset \mathbb{R}^{n}$, let $u^{\varepsilon}$, $e^{\varepsilon}, p^{\varepsilon}$ and $\sigma^{\varepsilon}$ be solutions to the $\varepsilon$-problem on $\mathcal{T}$ for $f=0$ and boundary data $w^{\varepsilon}=\xi^{\varepsilon} \cdot x+a^{\varepsilon}$, where $\xi^{\varepsilon} \rightarrow \xi$ weakly in $H^{1}\left(0, T ; \mathbb{R}_{s}^{n \times n}\right)$, $a^{\varepsilon} \rightarrow a$ weakly in $H^{1}\left(0, T ; \mathbb{R}^{n}\right)$. Then, as $\varepsilon \rightarrow 0$, for almost every $t \in[0, T]$ :

$$
f_{\mathcal{T}} \sigma^{\varepsilon}(t) \rightarrow \Sigma(\xi, t), \quad f_{\mathcal{T}} p^{\varepsilon}(t) \rightarrow \Pi(\xi, t) .
$$

Proof. The convergence (4.5) is a direct consequence of an energy estimate. Let $\tilde{u}^{\varepsilon}$, $\tilde{e}^{\varepsilon}, \tilde{p}^{\varepsilon}$ and $\tilde{\sigma}^{\varepsilon}$ be solutions to the $\varepsilon$-problem on $\mathcal{T} \subset \mathbb{R}^{n}$ with the right hand side $f=0$ and the limit boundary data $w(t)=\xi(t) \cdot x+a(t)$. The equation for $\tilde{u}^{\varepsilon}-u^{\varepsilon}$ can be multiplied with $\partial_{t}\left(\left(\tilde{u}^{\varepsilon}-w\right)-\left(u^{\varepsilon}-w^{\varepsilon}\right)\right)$ to obtain the energy estimate

$$
\left\|\nabla\left(u^{\varepsilon}-\tilde{u}^{\varepsilon}\right)\right\|_{\mathcal{V}^{0, \infty}}+\left\|\left(\sigma^{\varepsilon}-\tilde{\sigma}^{\varepsilon}\right)\right\|_{\mathcal{V}^{0, \infty}}+\left\|\left(e^{\varepsilon}-\tilde{e}^{\varepsilon}\right)\right\|_{\mathcal{V}^{0, \infty}}+\left\|\left(p^{\varepsilon}-\tilde{p}^{\varepsilon}\right)\right\|_{\mathcal{V}^{0, \infty}} \rightarrow 0
$$

as $\varepsilon \rightarrow 0$ in the space $\mathcal{V}^{0, \infty}=L^{\infty}\left(0, T ; L^{2}\left(\mathcal{T} ; \mathbb{R}^{n \times n}\right)\right)$. The convergence of the $\tilde{\sigma}^{\varepsilon}$ - and $\tilde{p}^{\varepsilon}$-averages provides (4.5).

The following theorem is our central stabilization result: The oscillatory solutions in a fixed simplex $\mathcal{T}$ converge necessarily to affine limit functions as $\varepsilon \rightarrow 0$. While the averaging property only demands convergence of the stress average over the simplex, we now find that, indeed, the stress converges weakly to a constant function (constant on $\mathcal{T}$, for every $t$ ).

Theorem 4.3 (Stabilization). Let the coefficients $C_{\varepsilon}, B_{\varepsilon}$ and $\Psi_{\varepsilon}$ allow averaging in the sense of Definition 1.3. For a simplex $\mathcal{T} \subset \mathbb{R}^{n}$, let $u^{\varepsilon}, e^{\varepsilon}, p^{\varepsilon}$ and $\sigma^{\varepsilon}$ be solutions to the $\varepsilon$-problem on $\mathcal{T}$ for $f=0$ and boundary data $w(x, t)=\xi(t) \cdot x$, where $\xi$ is in $H^{1}\left(0, T ; \mathbb{R}_{s}^{n \times n}\right)$. Then, as $\varepsilon \rightarrow 0$, there holds

$$
\begin{aligned}
& u^{\varepsilon} \rightarrow w \quad \text { weakly in } H^{1}\left(0, T ; H^{1}(\mathcal{T})\right) \\
& \sigma^{\varepsilon} \rightarrow \Sigma(\xi) \text { and } p^{\varepsilon} \rightarrow \Pi(\xi) \quad \text { weakly in } L^{2}\left(0, T ; L^{2}(\mathcal{T})\right) .
\end{aligned}
$$

Proof. The proof is similar to the proof of Proposition 2.8 in [20]: The simplex $\mathcal{T}$ now plays the role of the domain $Q$, and we can consider a triangulation of $\mathcal{T}$ with a fine grid. We can therefore exploit the approximation results that are obtained so far.

Step 1: Construction of auxiliary functions. We can extract a weakly convergent subsequence and set $u$ to be a weak limit of the original sequence $u^{\varepsilon}$ in $H^{1}\left(0, T ; H^{1}(\mathcal{T})\right)$.

For a fixed sequence $h \searrow 0$ and a fixed sequence of time discretizations, we make use of Corollary 2.2 and choose a polygonal domain $\mathcal{T}_{h} \subset \mathcal{T}$ with a triangulation $\mathbb{S}_{h}:=\left\{\mathcal{S}_{k}\right\}_{k \in \Lambda_{h}}$ such that $\mathbb{S}_{h}$ is an adapted grid for $u^{\varepsilon}$. On this grid, let $u_{h}^{\varepsilon}$ be a solution to the needle problem (see Definition 2.6). We use the right hand side $f=0$ and the affine boundary condition $w$ (together with $w_{h}=w$ ).

The family of needle problem solutions are bounded. We can therefore select a subsequence $\varepsilon \rightarrow 0$ and find, for limit functions $u_{h}$, the convergence $u_{h}^{\varepsilon} \rightarrow u_{h}$ weakly in $H^{1}\left(0, T ; H^{1}(\mathcal{T})\right)$. We recall that the functions $u_{h}^{\varepsilon}$ and $u_{h}$ are piecewise affine functions on $\partial \mathcal{S}_{k}$ for all $k$, but they are not necessarily affine in the single simplex $\mathcal{S}_{k}$.

Finally, for any $\varepsilon>0$ and $h>0$, we consider the piecewise affine interpolations $\bar{u}_{h}^{\varepsilon}:=\mathcal{F}_{h}^{w}\left(u_{h}^{\varepsilon}\right)$ of the needle problem solutions. Choosing a further subsequence if necessary, these functions are weakly convergent, we denote their weak limits by $\bar{u}_{h}$. The limit functions are affine in each simplex $\mathcal{S}_{k}$. 
Step 2: Characterization of $\bar{u}_{h}$. We investigate further the piecewise affine functions $\bar{u}_{h}^{\varepsilon}$ with the aim of showing $\bar{u}_{h}=w$. We use the abbreviations $\xi_{k}^{\varepsilon}:=\left.\nabla^{s} \bar{u}_{h}^{\varepsilon}\right|_{\mathcal{S}_{k}} \rightarrow$ $\left.\nabla^{s} \bar{u}_{h}\right|_{\mathcal{S}_{k}}=: \xi_{k}$ in $H^{1}((0, T))$ and find, using Lemma 4.2 , as $\varepsilon \rightarrow 0$ :

$$
f_{\mathcal{S}_{k}} \sigma^{\varepsilon}(t) \rightarrow \Sigma\left(\xi_{k}, t\right), \quad f_{\mathcal{S}_{k}} p^{\varepsilon}(t) \rightarrow \Pi\left(\xi_{k}, t\right) .
$$

We now choose an arbitrary test function $\phi \in L^{2}\left(0, T ; Y_{h}\right)$. Since $\nabla \phi$ is constant in space on any $\mathcal{S}_{k}$, and since $\left.\phi\right|_{\mathcal{T} \backslash \mathcal{T}_{h}} \equiv 0$, we obtain for arbitrary $t$, as $\varepsilon \rightarrow 0$,

$$
0=\int_{\mathcal{T}} \sigma_{h}^{\varepsilon}: \nabla \phi=\sum_{k} \int_{\mathcal{S}_{k}} \sigma_{h}^{\varepsilon}: \nabla \phi \rightarrow \sum_{k} \int_{\mathcal{S}_{k}} \Sigma\left(\xi_{k}\right): \nabla \phi=\int_{\mathcal{T}} \Sigma\left(\nabla^{s} \bar{u}_{h}\right): \nabla \phi .
$$

Lemma 3.2 shows that solutions in $w+H^{1}\left(0, T ; Y_{h}\right)$ to $(4.9)$ are uniquely determined. On the other hand, the affine function $w$ is a solution itself, since $\Sigma(\nabla w)$ is a constant function. We have therefore obtained $\bar{u}_{h}=w$.

Step 3: Comparison of limits. We apply the needle problem comparison result of Proposition 4.1 together with weak lower semicontinuity of norms to find

$$
\lim _{h \rightarrow 0}\left\|u-u_{h}\right\|_{L^{\infty}\left(0, T ; H^{1}(\mathcal{T})\right)} \leq \lim _{h \rightarrow 0} \limsup _{\varepsilon \rightarrow 0}\left\|u^{\varepsilon}-u_{h}^{\varepsilon}\right\|_{L^{\infty}\left(0, T ; H^{1}(\mathcal{T})\right)}=0 .
$$

The only (distributional) limit of $u_{h}$ is therefore $u$; in particular, we find $u_{h} \rightarrow u$ weakly in $H^{1}\left(0, T ; H^{1}(\mathcal{T})\right)$ as $h \rightarrow 0$.

The weak continuity of $\mathcal{F}_{h}^{w}$, Lemma 2.4 item (ii), provides, as $\varepsilon \rightarrow 0$,

$$
\mathcal{F}_{h}^{w}\left(u_{h}\right)\left\llcorner\mathcal{F}_{h}^{w}\left(u_{h}^{\varepsilon}\right)=\bar{u}_{h}^{\varepsilon} \rightarrow \bar{u}_{h}=w .\right.
$$

On the other hand, $u_{h} \rightarrow u$ weakly in $H^{1}\left(0, T ; H^{1}(\mathcal{T})\right)$ allows to apply Lemma 2.4, item (iii). We conclude that $\mathcal{F}_{h}^{w}\left(u_{h}\right)$ converges, for $h \rightarrow 0$, to $u$. This provides $u=w$ and hence (4.7).

Step 4: Convergence of stress and strain. It remains to prove (4.8). We select weakly convergent subsequences and consider $e^{\varepsilon} \rightarrow e, p^{\varepsilon} \rightarrow p$ and $\sigma^{\varepsilon} \rightarrow \sigma$ as well as $e_{h}^{\varepsilon} \rightarrow e_{h}, p_{h}^{\varepsilon} \rightarrow p_{h}$ and $\sigma_{h}^{\varepsilon} \rightarrow \sigma_{h}$ in $H^{1}\left(0, T ; L^{2}(\mathcal{T})\right)$. From the weak lower semicontinuity of norms and Proposition 4.1, we know

$$
\begin{aligned}
\lim _{h \rightarrow 0}\left(\left\|\nabla^{s} u-\nabla^{s} u_{h}\right\|_{\mathcal{V}^{0}}+\left\|\sigma-\sigma_{h}\right\|_{\mathcal{V}^{0}}+\left\|p-p_{h}\right\|_{\mathcal{V}^{0}}\right) \leq \\
\lim _{h \rightarrow 0} \limsup _{\varepsilon \rightarrow 0}\left(\left\|\nabla^{s} u^{\varepsilon}-\nabla^{s} u_{h}^{\varepsilon}\right\|_{\mathcal{V}^{0}}+\left\|\sigma^{\varepsilon}-\sigma_{h}^{\varepsilon}\right\|_{\mathcal{V}^{0}}+\left\|p^{\varepsilon}-p_{h}^{\varepsilon}\right\|_{\mathcal{V}^{0}}\right)=0,
\end{aligned}
$$

where the norm is that of $\mathcal{V}^{0}=L^{2}\left(0, T ; L^{2}(\mathcal{T})\right)$.

With this information at hand, we will be able to characterize limits. We choose an arbitrary function $\varphi \in C\left([0, T] ; C_{c}^{1}(\mathcal{T})^{n}\right)$ and choose a sequence of piecewise constant functions $\varphi_{h} \in L^{2}\left(0, T ; L^{2}(Q)\right)$ (constant on $\mathcal{S}_{k}$ for every $k \in \Lambda_{h}$ ), such that $\left\|\varphi-\varphi_{h}\right\|_{L^{2}\left(0, T ; L^{2}(\mathcal{T})\right)} \rightarrow 0$ as $h \rightarrow 0$. We calculate, for $\varepsilon \rightarrow 0$,

$$
\begin{aligned}
\int_{\mathcal{T}} \sigma_{h}: \varphi_{h} & \leftarrow \int_{\mathcal{T}} \sigma_{h}^{\varepsilon}: \varphi_{h}=\sum_{k} \int_{\mathcal{S}_{k}} \sigma_{h}^{\varepsilon}: \varphi_{h} \\
& \rightarrow \sum_{k} \int_{\mathcal{S}_{k}} \Sigma\left(\xi_{k}\right): \varphi_{h}=\int_{\mathcal{T}} \Sigma\left(\nabla^{s} \bar{u}_{h}\right): \varphi_{h}=\int_{\mathcal{T}} \Sigma\left(\nabla^{s} w\right): \varphi_{h}=\int_{\mathcal{T}} \Sigma(\xi): \varphi_{h} .
\end{aligned}
$$

The strong convergences $\sigma_{h} \rightarrow \sigma$ and $\varphi_{h} \rightarrow \varphi$ provide, since $\varphi$ was arbitrary, the relation $\sigma=\Sigma(\xi)$. An analogous calculation shows $p=\Pi(\xi)$, which verifies (4.8). 
Similar to our procedure in Lemma 4.2, we now generalize the result to a situation with $\varepsilon$-dependent boundary data.

Corollary 4.4 (Stabilization for sequences of boundary data). Let the coefficients $C_{\varepsilon}$, $B_{\varepsilon}$ and $\Psi_{\varepsilon}$ allow averaging in the sense of Definition 1.3. For a simplex $\mathcal{T} \subset \mathbb{R}^{n}$, let $u^{\varepsilon}, e^{\varepsilon}, p^{\varepsilon}$ and $\sigma^{\varepsilon}$ be solutions to the $\varepsilon$-problem (1.1) with $f=0$ and $w^{\varepsilon}(x, t)=\xi^{\varepsilon}(t) \cdot x+$ $a^{\varepsilon}(t)$, where $\xi^{\varepsilon} \in H^{1}\left(0, T ; \mathbb{R}_{s}^{n \times n}\right)$ and $a^{\varepsilon} \in H^{1}\left(0, T ; \mathbb{R}^{n}\right)$. We assume that $\xi^{\varepsilon} \rightarrow \xi$ weakly in $H^{1}\left(0, T ; \mathbb{R}_{s}^{n \times n}\right)$ and $a^{\varepsilon} \longrightarrow a$ weakly in $H^{1}\left(0, T ; \mathbb{R}^{n}\right)$, and set $w(x, t)=\xi(t) \cdot x+a(t)$. Then, as $\varepsilon \rightarrow 0$,

$$
\begin{gathered}
u^{\varepsilon} \rightarrow w(x, t)=\xi(t) \cdot x+a(t) \text { weakly in } H^{1}\left(0, T ; H^{1}(\mathcal{T})\right), \\
p^{\varepsilon}(.) \rightarrow \Pi\left(\nabla^{s} w, .\right), \sigma^{\varepsilon}(.) \rightarrow \Sigma\left(\nabla^{s} w, .\right) \text { weakly in } L^{2}\left(0, T ; L^{2}(\mathcal{T})\right) .
\end{gathered}
$$

Proof. Let $\tilde{u}^{\varepsilon}, \tilde{e}^{\varepsilon}, \tilde{p}^{\varepsilon}$ and $\tilde{\sigma}^{\varepsilon}$ be solutions to the $\varepsilon$-problem with $f=0$ and $w=\xi \cdot x+a$. Due to our a priori estimates and the stabilization result of Theorem 4.3 it suffices to prove

$$
\lim _{\varepsilon \rightarrow 0}\left(\left\|\nabla^{s} u^{\varepsilon}-\nabla^{s} \tilde{u}^{\varepsilon}\right\|_{\mathcal{V}^{0}}+\left\|\sigma^{\varepsilon}-\tilde{\sigma}^{\varepsilon}\right\|_{\mathcal{V}^{0}}+\left\|p^{\varepsilon}-\tilde{p}^{\varepsilon}\right\|_{\mathcal{V}^{0}}\right)=0
$$

where the norm is $\mathcal{V}^{0}=L^{2}\left(0, T ; L^{2}(\mathcal{T})\right)$.

This energy type estimate is obtained as in other proofs with a testing procedure (see, e.g. the proof of Lemma 4.2): We use equation (1.8) ${ }_{1}$ for both $u^{\varepsilon}$ and $\tilde{u}^{\varepsilon}$, substract the two equations, and use $\partial_{t}\left(u^{\varepsilon}-w^{\varepsilon}\right)-\partial_{t}\left(\tilde{u}^{\varepsilon}-w\right)$ as a test function. With the monotonicity of the flow rule, we obtain (4.12).

\subsection{Needle problem and discretized limit problem}

We can now use the stabilization result of Corollary 4.4 to derive the remaining smallness result, the horizontal arrow in (2.1): We show that the solutions to the needle problem converge to the solution of the discretized limit problem.

Proposition 4.5. Let the domain $Q \subset \mathbb{R}^{n}$, the data $w$ and $f$, and the coefficients be as in (1.2)-(1.5). We assume that the coefficients allow averaging in sense of Definition 1.3 with causal operators $\Sigma$ and $\Pi$.

Let $\left(u_{h}^{\varepsilon}, e_{h}^{\varepsilon}, p_{h}^{\varepsilon}, \sigma_{h}^{\varepsilon}\right)$ be solutions to the needle problem (2.16)-(2.18). Then, as $\varepsilon \rightarrow 0$, we find $u_{h}^{\varepsilon} \rightarrow u_{h}$ weakly in $H^{1}\left(0, T ; H^{1}(Q)\right)$ and $\sigma_{h}^{\varepsilon} \rightarrow \Sigma\left(\nabla u_{h}\right), p_{h}^{\varepsilon} \rightarrow \Pi\left(\nabla u_{h}\right)$ weakly in the space $H^{1}\left(0, T ; L^{2}\left(Q, \mathbb{R}_{s}^{n \times n}\right)\right)$, where $u_{h} \in w_{h}+Y_{h}$ is the unique weak solution to the discretized problem (3.7),

$$
\int_{0}^{T} \int_{Q_{h}} \Sigma\left(\nabla^{s} u_{h}\right): \nabla \varphi=\int_{0}^{T} \int_{Q_{h}} f \cdot \varphi \quad \forall \varphi \in L^{2}\left(0, T ; Y_{h}\right) .
$$

Proof. The a priori estimates for the needle problem solutions $u_{h}^{\varepsilon}$ allow to extract a subsequence $\varepsilon \rightarrow 0$ such that $u_{h}^{\varepsilon} \rightarrow \tilde{u}_{h}$ in $H^{1}\left(0, T ; H^{1}(Q)\right)$ for some limit function $\tilde{u}_{h}$.

The functions $u_{h}^{\varepsilon} \in w_{h}+L^{2}\left(0, T ; \mathcal{N}_{h}\right)$ are affine on the boundaries of each simplex $\mathcal{T}_{k}$. For fixed $k, \varepsilon$, and $h$, we write this affine function as $w_{k}^{\varepsilon}(x, t)=\xi_{k}^{\varepsilon}(t) \cdot x+a_{k}^{\varepsilon}(t)$ for $x \in \mathcal{T}_{k}$. For a further subsequence, we find an affine function $w_{k}(x, t)=\xi_{k}(t) \cdot x+a_{k}(t)$ such that $w_{k}^{\varepsilon} \rightarrow w_{k}$ weakly in $H^{1}\left(0, T ; H^{1}\left(\mathcal{T}_{k}\right)\right)$. Considering a fixed simplex $\mathcal{T}_{k}$, we can apply Corollary 4.4. We obtain

$$
\left.u_{h}^{\varepsilon}\right|_{\mathcal{T}_{k}} \rightarrow w_{k},\left.\quad p_{h}^{\varepsilon}\right|_{\mathcal{T}_{k}} \rightarrow \Pi\left(\nabla^{s} w_{k}, .\right),\left.\quad \sigma_{h}^{\varepsilon}\right|_{\mathcal{T}_{k}} \rightarrow \Sigma\left(\nabla^{s} w_{k}, .\right),
$$


weakly in $H^{1}\left(0, T ; H^{1}\left(\mathcal{T}_{k}\right)\right)$ and $H^{1}\left(0, T ; L^{2}\left(\mathcal{T}_{k}\right)\right)$, respectively. Since the number of simplices is finite in this consideration, the above convergence implies $\tilde{u}_{h} \in L^{2}\left(0, T ; Y_{h}\right)$ with $\left.\tilde{u}_{h}\right|_{\mathcal{T}_{k}}=w_{k}$, and

$$
u_{h}^{\varepsilon} \rightarrow \tilde{u}_{h}, \quad p_{h}^{\varepsilon} \rightarrow \Pi\left(\nabla^{s} \tilde{u}_{h}, t\right), \quad \sigma_{h}^{\varepsilon} \rightarrow \Sigma\left(\nabla^{s} \tilde{u}_{h}, t\right),
$$

as $\varepsilon \rightarrow 0$, weakly in $H^{1}\left(0, T ; H^{1}(Q)\right)$ and $H^{1}\left(0, T ; L^{2}(Q)\right)$, respectively.

We next use an arbitrary test function $\varphi \in L^{2}\left(0, T ; Y_{h}\right)$. The needle problem relation (2.16) allows to calculate

$$
\int_{0}^{T} \int_{Q} f \cdot \varphi \stackrel{(2.12)}{=} \int_{0}^{T} \int_{\Gamma_{h}} g_{h} \cdot \varphi=\int_{0}^{T} \int_{Q} \sigma_{h}^{\varepsilon}: \nabla \varphi \rightarrow \int_{0}^{T} \int_{Q} \Sigma\left(\nabla^{s} \tilde{u}_{h}\right): \nabla \varphi .
$$

We obtain that $\tilde{u}_{h}$ solves (4.13). Since the solution to this relation is unique by Lemma 3.2 , we find $\tilde{u}_{h}=u_{h}$. The convergences of $u_{h}^{\varepsilon}$ to $\tilde{u}_{h}$ and $\sigma_{h}^{\varepsilon}$ to $\Sigma\left(\nabla^{s} \tilde{u}_{h}\right)$ yield the claim.

\subsection{Proof of Theorem 1.6}

We are given a sequence $\varepsilon=\varepsilon_{j} \rightarrow 0$ and a sequence of solutions $u^{\varepsilon}$ of the original problem. For a sequence $h \rightarrow 0$ and a sequence of time discretizations $0 \leq t_{0}^{h}<t_{1}^{h}<$ $\ldots<t_{K}^{h} \leq T$ with $\max _{k}\left|t_{k+1}^{h}-t_{k}^{h}\right| \leq h$ we can choose a sequence of grids $\mathbb{T}_{h}$ that is adapted to $u^{\varepsilon}$ in the sense of Corollary 2.2.

For each $h>0$ and $\varepsilon>0$ we consider the unique solution $u_{h}^{\varepsilon} \in w_{h}+H^{1}\left(0, T ; \mathcal{N}_{h}\right)$, $e_{h}^{\varepsilon}, p_{h}^{\varepsilon}, \sigma_{h}^{\varepsilon} \in H^{1}\left(0, T ; L^{2}\left(Q_{h}\right)\right)$ to the needle problem of Definition 2.6. Proposition 4.5 implies that $u_{h}^{\varepsilon} \rightarrow u_{h}$ weakly in $w_{h}+H^{1}\left(0, T ; Y_{h}\right)$ as $\varepsilon \rightarrow 0$, where $u_{h}$ is the unique solution to the discrete problem (4.13).

We next want to apply Proposition 3.4. The proposition is applicable: (i) The admissibility assumption of Definition 1.5 guarantees the existence of a solution $u$ to the effective problem. (ii) Proposition 4.5 guarantees the existence of a solution $u_{h}$ to the discretized effective problem. Proposition 3.4 yields $u_{h} \rightarrow u$ weakly in $H^{1}\left(0, T ; H^{1}(Q)\right)$ as $h \rightarrow 0$, where $u$ is the solution of the effective system. Proposition 4.1 provides smallness of $u^{\varepsilon}-u_{h}^{\varepsilon}$. With a triangle inequality we obtain

$$
\begin{aligned}
& \lim _{\varepsilon \rightarrow 0}\left\|u^{\varepsilon}-u\right\|_{L^{2}\left(Q_{T}\right)} \\
& \quad \leq \liminf _{h \rightarrow 0} \limsup _{\varepsilon \rightarrow 0}\left(\left\|u^{\varepsilon}-u_{h}^{\varepsilon}\right\|_{L^{2}\left(Q_{T}\right)}+\left\|u_{h}^{\varepsilon}-u_{h}\right\|_{L^{2}\left(Q_{T}\right)}+\left\|u_{h}-u\right\|_{L^{2}\left(Q_{T}\right)}\right)=0 .
\end{aligned}
$$

This characterizes every weak limit of (subsequences of) $u^{\varepsilon}$ and, therefore, provides the weak convergence (1.15) of Theorem 1.6.

We now analyze the sequence of stresses and (plastic) strains. The needle problem solution $u_{h}^{\varepsilon}$ is accompanied by $\sigma_{h}^{\varepsilon}$ and $p_{h}^{\varepsilon}$. Proposition 4.5 provides $\sigma_{h}^{\varepsilon} \rightarrow \Sigma\left(\nabla^{s} u_{h}\right)$ and $p_{h}^{\varepsilon} \rightarrow \Pi\left(\nabla^{s} u_{h}\right)$ weakly as $\varepsilon \rightarrow 0$. Proposition 3.4 yields $\Sigma\left(\nabla^{s} u_{h}\right) \rightarrow \Sigma\left(\nabla^{s} u\right)$ and $\Pi\left(\nabla^{s} u_{h}\right) \rightarrow \Pi\left(\nabla^{s} u\right)$ strongly in $L^{\infty}\left(0, T ; L^{2}(Q)\right)$ as $h \rightarrow 0$. Finally, Proposition 4.1 compares $\sigma_{h}^{\varepsilon}$ with $\sigma^{\varepsilon}$ and $p_{h}^{\varepsilon}$ with $p^{\varepsilon}$.

Due to the a priori estimate (1.10) we find a subsequence $\varepsilon \rightarrow 0$ and limits $\sigma$ and $p$ such that $\sigma^{\varepsilon} \rightarrow \sigma$ and $p^{\varepsilon} \rightarrow p$ weakly in $H^{1}\left(0, T ; L^{2}\left(Q, \mathbb{R}_{s}^{n \times n}\right)\right)$. In order to characterize 
these limits we calculate, for arbitrary $\varphi \in L^{2}\left(0, T ; L^{2}\left(Q ; \mathbb{R}^{n \times n}\right)\right)$,

$$
\begin{aligned}
\int_{0}^{T} \int_{Q} \sigma: \varphi & =\lim _{\varepsilon \rightarrow 0} \int_{0}^{T} \int_{Q} \sigma^{\varepsilon}: \varphi=\lim _{h \rightarrow 0} \lim _{\varepsilon \rightarrow 0} \int_{0}^{T} \int_{Q} \sigma_{h}^{\varepsilon}: \varphi \\
& =\lim _{h \rightarrow 0} \int_{0}^{T} \int_{Q} \Sigma\left(\nabla^{s} u_{h}\right): \varphi=\int_{0}^{T} \int_{Q} \Sigma\left(\nabla^{s} u\right): \varphi .
\end{aligned}
$$

This shows $\sigma=\Sigma\left(\nabla^{s} u\right)$. A similar calculation shows $p^{\varepsilon} \rightarrow \Pi\left(\nabla^{s} u\right)$ weakly in the space $H^{1}\left(0, T ; L^{2}\left(Q ; \mathbb{R}^{n \times n}\right)\right)$ as $\varepsilon \rightarrow 0$. This provides (1.16) and concludes the proof of our main result, Theorem 1.6.

\section{A On the proof of Theorem 1.2}

We present here only the (formal) a priori estimates. On the basis of the a priori estimates for the discretized system, the construction of a solution is performed in a standard way with a Galerkin scheme (as e.g. in [19]). In the following, we therefore suppose that a sufficiently regular solution $u^{\varepsilon}$ to the $\varepsilon$-problem (1.8) is given. Our aim is to derive the estimate (1.10).

We obtain energy type estimates with a testing procedure. In a first step, we use $\chi_{[0, t]} \partial_{t}\left(u^{\varepsilon}-w\right)$ as a test function in the original equation $-\nabla \cdot \sigma^{\varepsilon}=f$; here $\chi_{[0, t]}$ is the characteristic function of the interval $[0, t]$ for $t<T$. In a second step, we differentiate the equation with respect to time and use $\partial_{t}\left(u^{\varepsilon}-w\right)$ as test function.

In what follows, we write $\|\cdot\|_{L^{2} L^{2}}$ for the norm in $L^{2}\left(0, T ; L^{2}(Q)\right)$ and $\|\cdot\|_{H^{1} L^{2}}$ for the norm in $H^{1}\left(0, T ; L^{2}(Q)\right)$; other norms are abbreviated in the analogous way, we use $\|\cdot\|_{L^{2} H^{1}}$ and $\|\cdot\|_{H^{1} H^{1}}$.

The test function $\chi_{[0, t]} \partial_{t}\left(u^{\varepsilon}-w\right)$ provides

$$
\begin{aligned}
\int_{0}^{t} \int_{Q} f \cdot \partial_{t}\left(u^{\varepsilon}-w\right)+\int_{0}^{t} \int_{Q} \sigma^{\varepsilon}: \nabla^{s} \partial_{t} w=\int_{0}^{t} \int_{Q} \sigma^{\varepsilon}: \nabla^{s} \partial_{t} u^{\varepsilon} \\
\quad=\int_{0}^{t} \int_{Q} \sigma^{\varepsilon}: \partial_{t}\left(C_{\varepsilon} \sigma^{\varepsilon}+p^{\varepsilon}\right) \\
\quad=\int_{0}^{t} \int_{Q}\left(\frac{1}{2} \frac{d}{d t}\left|\sigma^{\varepsilon}\right|_{C_{\varepsilon}}^{2}+\left(\sigma^{\varepsilon}-B_{\varepsilon} p^{\varepsilon}\right): \partial_{t} p^{\varepsilon}+\left(B_{\varepsilon} p^{\varepsilon}\right): \partial_{t} p^{\varepsilon}\right) \\
\quad=\int_{0}^{t} \int_{Q}\left(\frac{1}{2} \frac{d}{d t}\left(\left|\sigma^{\varepsilon}\right|_{C_{\varepsilon}}^{2}+\left|p^{\varepsilon}\right|_{B_{\varepsilon}}^{2}\right)+\left(\sigma^{\varepsilon}-B_{\varepsilon} p^{\varepsilon}\right): \partial_{t} p^{\varepsilon}\right) \\
\quad \stackrel{(*)}{=} \int_{0}^{t} \int_{Q}\left(\frac{1}{2} \frac{d}{d t}\left(\left|\sigma^{\varepsilon}\right|_{C_{\varepsilon}}^{2}+\left|p^{\varepsilon}\right|_{B_{\varepsilon}}^{2}\right)+\Psi_{\varepsilon}^{*}\left(\partial_{t} p^{\varepsilon}\right)+\Psi_{\varepsilon}\left(\sigma^{\varepsilon}-B_{\varepsilon} p^{\varepsilon}\right)\right)
\end{aligned}
$$

where in $(*)$ we used that for $\Psi_{\varepsilon}$ and its conjugate $\Psi_{\varepsilon}^{*}$ holds

$$
\left(\sigma^{\varepsilon}-B_{\varepsilon} p^{\varepsilon}\right): \partial_{t} p^{\varepsilon}=\Psi_{\varepsilon}^{*}\left(\partial_{t} p^{\varepsilon}\right)+\Psi_{\varepsilon}\left(\sigma^{\varepsilon}-B_{\varepsilon} p^{\varepsilon}\right)
$$

because of $\partial_{t} p^{\varepsilon} \in \partial \Psi_{\varepsilon}\left(\sigma^{\varepsilon}-B_{\varepsilon} p^{\varepsilon}\right)$. Since $t \leq T$ was arbitrary, we obtain from (A.1)

$$
\begin{aligned}
\sup _{t \in[0, T]} & \left\|\sigma^{\varepsilon}(t)\right\|_{L^{2}(Q)}^{2}+\sup _{t \in[0, T]}\left\|p^{\varepsilon}(t)\right\|_{L^{2}(Q)}^{2} \leq\|f\|_{L^{2} L^{2}}\left\|\partial_{t} w\right\|_{L^{2} L^{2}} \\
& +\|f\|_{L^{2} L^{2}}\left\|\partial_{t} u^{\varepsilon}\right\|_{L^{2} L^{2}}+\|w\|_{H^{1} H^{1}}^{2}+\left\|\sigma^{\varepsilon}\right\|_{L^{2} L^{2}}^{2}+\left\|\sigma^{\varepsilon}(0)\right\|_{L^{2}(Q)}^{2}
\end{aligned}
$$


where we used $\left\|p^{\varepsilon}(0)\right\|_{L^{2}(Q)}^{2}=0$ and the fact that $\Psi_{\varepsilon}$ and $\Psi_{\varepsilon}^{*}$ are nonnegative.

In order to obtain an estimate for $\partial_{t} \sigma^{\varepsilon}, \partial_{t} p^{\varepsilon}, \partial_{t} \nabla u^{\varepsilon}$, and $\partial_{t} u^{\varepsilon}$ we apply the time derivative $\partial_{t}$ to the equation $-\nabla \cdot \sigma^{\varepsilon}=f$. We use the test function $\partial_{t}\left(u^{\varepsilon}-w\right)$ and integrate over $[0, T] \times Q$. With an integration by parts we obtain

$$
\begin{aligned}
\int_{0}^{T} & \int_{Q} \partial_{t} f \cdot \partial_{t}\left(u^{\varepsilon}-w\right)+\int_{0}^{T} \int_{Q} \partial_{t} \sigma^{\varepsilon}: \nabla^{s} \partial_{t} w=\int_{0}^{T} \int_{Q} \partial_{t} \sigma^{\varepsilon}: \nabla^{s} \partial_{t} u^{\varepsilon} \\
& =\int_{0}^{T} \int_{Q} \partial_{t} \sigma^{\varepsilon}: \partial_{t}\left(C_{\varepsilon} \sigma^{\varepsilon}+p^{\varepsilon}\right)=\int_{0}^{T} \int_{Q}\left(\left|\partial_{t} \sigma^{\varepsilon}\right|_{C_{\varepsilon}}^{2}+\partial_{t} \sigma^{\varepsilon}: \partial_{t} p^{\varepsilon}\right) \\
& =\int_{0}^{T} \int_{Q}\left(\left|\partial_{t} \sigma^{\varepsilon}\right|_{C_{\varepsilon}}^{2}+\left|\partial_{t} p^{\varepsilon}\right|_{B_{\varepsilon}}^{2}+\partial_{t}\left[\sigma^{\varepsilon}-B_{\varepsilon} p^{\varepsilon}\right]: \partial_{t} p^{\varepsilon}\right) \\
& =\int_{0}^{T} \int_{Q}\left(\left|\partial_{t} \sigma^{\varepsilon}\right|_{C_{\varepsilon}}^{2}+\left|\partial_{t} p^{\varepsilon}\right|_{B_{\varepsilon}}^{2}+\frac{d}{d t} \Psi_{\varepsilon}\left(\sigma^{\varepsilon}-B_{\varepsilon} p^{\varepsilon}\right)\right) .
\end{aligned}
$$

By nonnegativity of $\Psi_{\varepsilon}\left(\sigma^{\varepsilon}(t)-B_{\varepsilon} p^{\varepsilon}(t)\right)$ we obtain

$$
\begin{aligned}
\left\|\partial_{t} \sigma^{\varepsilon}\right\|_{L^{2} L^{2}}^{2} & +\left\|\partial_{t} p^{\varepsilon}\right\|_{L^{2} L^{2}}^{2} \leq\|f\|_{H^{1} L^{2}}\left\|\partial_{t} w\right\|_{L^{2} L^{2}} \\
& +\|f\|_{H^{1} L^{2}}\left\|\partial_{t} u^{\varepsilon}\right\|_{L^{2} L^{2}}+\|w\|_{H^{1} H^{1}}^{2}+\frac{1}{2}\left\|\partial_{t} \sigma^{\varepsilon}\right\|_{L^{2} L^{2}}^{2}+\left\|\Psi_{\varepsilon}\left(\sigma^{\varepsilon}(0)\right)\right\|_{L^{2}}
\end{aligned}
$$

This provides essentially a bound for the norms of (1.10). The variables $\partial_{t} \sigma^{\varepsilon}$ and $\partial_{t} p^{\varepsilon}$ control $\partial_{t} e^{\varepsilon}$ by the additive decomposition of the strain. As a consequence, Korn's inequality allows to control $\partial_{t} \nabla u^{\varepsilon}$. Formally, we can absorb the factor $\left\|\partial_{t} u^{\varepsilon}\right\|_{L^{2} L^{2}}^{2}$ of (A.2) and (A.4) with the help of

$$
\begin{aligned}
& \left\|\partial_{t} u^{\varepsilon}(t)\right\|_{L^{2}(Q)} \leq\left\|\partial_{t} u^{\varepsilon}(t)\right\|_{H^{1}(Q)} \leq C\left(\left\|\partial_{t} \nabla^{s} u^{\varepsilon}(t)\right\|_{L^{2}(Q)}+\left\|\partial_{t} w(t)\right\|_{H^{1}(Q)}\right) \\
& \quad \leq C\left(\left\|\partial_{t} p^{\varepsilon}(t)\right\|_{L^{2}(Q)}+\left\|\partial_{t} \sigma^{\varepsilon}(t)\right\|_{L^{2}(Q)}+\left\|\partial_{t} w(t)\right\|_{H^{1}(Q)}\right) \quad \forall t \in[0, T] .
\end{aligned}
$$

Young's inequality provides the a priori estimate (1.10) for $\partial_{t} \sigma^{\varepsilon}$ and $\partial_{t} p^{\varepsilon}$. The estimate on $u^{\varepsilon}$ follows from (A.5), the estimate for the original functions follows from (A.2).

\section{B Outline of the proof of Theorem 2.7}

Regularization, Galerkin scheme and existence. The proof follows the lines of the proof of Theorem 1.1. in [19]. The basic idea is to regularize the potential $\Psi_{\varepsilon}$ and to use a space discretization to construct approximate solutions. We approximate $\Psi_{\varepsilon}$ by its Yosida transform $\Psi_{\varepsilon, \delta}(\sigma):=\inf _{\xi}\left\{\Psi_{\varepsilon}(\xi)+\frac{|\sigma-\xi|}{\delta}\right\}$. We furthermore regularize $w_{h}$ to a function $w_{h, \delta} \in C^{1}\left([0, T] ; \tilde{Y}_{h}\right)$. Approximate solutions are constructed with finite element spaces: Let $\mathbb{T}_{h}=\left\{\mathcal{T}_{k}\right\}_{\Lambda_{h}}$ be the (fixed) grid with typical length scale $h$. For any $0<\eta<h$ let $\mathbb{T}_{h, \eta}=\left\{\mathcal{T}_{j}^{\eta}\right\}_{j \in \Lambda_{h, \eta}}$ be a refinement of $\mathcal{T}_{h}$ in the following sense: $\Lambda_{h, \eta}$ is a finite set for all $\eta$ and there holds

$$
\operatorname{diam} \mathcal{T}_{j}^{\eta} \leq \eta, \quad \mathcal{T}_{j}^{\eta} \cap \mathcal{T}_{k} \neq \emptyset \Rightarrow \mathcal{T}_{j}^{\eta} \subset \mathcal{T}_{k} \quad \forall j \in \Lambda_{h, \eta}
$$


Let

$$
\begin{aligned}
Y_{h, \eta} & :=\left\{\phi \in H_{0}^{1}(Q):\left.\phi\right|_{T_{j}^{\eta}} \text { is affine } \forall \mathcal{T}_{j}^{\eta} \in \mathbb{T}_{h, \eta}, \phi \equiv 0 \text { on } Q \backslash Q_{h}\right\}, \\
W_{h, \eta} & :=\left\{\phi \in L^{2}(Q):\left.\phi\right|_{T_{j}^{\eta}} \text { is constant } \forall \mathcal{T}_{j}^{\eta} \in \mathbb{T}_{h, \eta}, \phi \equiv 0 \text { on } Q \backslash Q_{h}\right\}, \\
\mathcal{P}_{h, \eta} & : L^{2}(Q) \rightarrow W_{h, \eta}, \quad \text { the } L^{2} \text {-orthogonal projection. }
\end{aligned}
$$

We look for solutions $u_{h, \eta, \delta}^{\varepsilon}=v_{h, \eta, \delta}^{\varepsilon}+w_{h, \delta}$ with $v_{h, \eta, \delta}^{\varepsilon} \in C^{1}\left(0, T ; Y_{h, \eta}\right)$ and $p_{h, \eta, \delta}^{\varepsilon}, e_{h, \eta, \delta}^{\varepsilon}$, $\sigma_{h, \eta, \delta}^{\varepsilon} \in C^{1}\left(0, T ; W_{h, \eta}\right)$ to the discrete system

$$
\begin{aligned}
\int_{Q} \sigma_{h, \eta, \delta}^{\varepsilon}(t): \nabla \varphi & =\int_{\Gamma_{h}} g_{h}(t) \cdot \varphi \quad \forall \varphi \in Y_{h, \eta}, \quad \forall t \in[0, T] \\
\nabla^{s} u_{h, \eta, \delta}^{\varepsilon} & =C_{\varepsilon} \sigma_{h, \eta, \delta}^{\varepsilon}+p_{h, \eta, \delta}^{\varepsilon} \\
\partial_{t} p_{h, \eta, \delta}^{\varepsilon} & =\partial \Psi_{\varepsilon, \eta, \delta}\left(\sigma_{h, \eta, \delta}^{\varepsilon}-B_{\varepsilon} p_{h, \eta, \delta}^{\varepsilon}\right)
\end{aligned}
$$

where $\Psi_{\varepsilon, \eta, \delta}(\sigma ; x)=\Psi_{\varepsilon, \delta}\left(\mathcal{P}_{h, \eta, \delta}(\sigma) ; \bar{x}_{\eta}\right)$, where $\bar{x}_{\eta}$ is a representative point in the $\eta$ size simplex that contains $x$. As in the proof of [19] Lemma 2.3, we can show that (B.1)-(B.3) has a unique solution $p_{h, \eta, \delta}^{\varepsilon}, u_{h, \eta, \delta}^{\varepsilon}$ and $\sigma_{h, \eta, \delta}^{\varepsilon}$. The derivation of a priori estimates follows the lines of Appendix A. As in [19], we can consider the joint limit $\eta \rightarrow 0, \delta \rightarrow 0$ and obtain weakly convergent subsequences $u_{h, \eta, \delta}^{\varepsilon} \rightarrow u_{h}^{\varepsilon}$, weakly in $H^{1}\left(0, T ; H^{1}(Q)\right)$ and $\sigma_{h, \eta, \delta}^{\varepsilon} \rightarrow \sigma_{h}^{\varepsilon}, p_{h, \eta, \delta}^{\varepsilon} \rightarrow p_{h}^{\varepsilon}$ weakly in $H^{1}\left(0, T ; L^{2}(Q)\right)$, such that $u_{h}^{\varepsilon}$, $\sigma_{h}^{\varepsilon}, p_{h}^{\varepsilon}$ satisfy (2.16)-(2.18) and the apriori estimate (2.20).

Uniqueness. In order to show uniqueness, we assume that we were given two solutions, $\left(u_{h, 1}^{\varepsilon}, \sigma_{h, 1}^{\varepsilon}, p_{h, 1}^{\varepsilon}\right)$ and $\left(u_{h, 2}^{\varepsilon}, \sigma_{h, 2}^{\varepsilon}, p_{h, 2}^{\varepsilon}\right)$. We use equation (2.16) for $u_{h, 1}^{\varepsilon}$ and $u_{h, 2}^{\varepsilon}$ and take the difference of the two equations. We introduce the difference $\left(\tilde{u}_{h}^{\varepsilon}, \tilde{\sigma}_{h}^{\varepsilon}, \tilde{p}_{h}^{\varepsilon}\right):=$ $\left(u_{h, 1}^{\varepsilon}-u_{h, 2}^{\varepsilon}, \sigma_{h, 1}^{\varepsilon}-\sigma_{h, 2}^{\varepsilon}, p_{h, 1}^{\varepsilon}-p_{h, 2}^{\varepsilon}\right)$ and insert $\partial_{t} \tilde{u}_{h}^{\varepsilon}$ as test function. Following the calculations of Appendix A, we obtain

$$
\begin{aligned}
&\left.\int_{\Gamma_{h}} g_{h} \tilde{u}_{h}^{\varepsilon}\right|_{0} ^{T}-\int_{0}^{T} \int_{\Gamma_{h}} \partial_{t} g_{h} \tilde{u}_{h}^{\varepsilon}=\int_{\Gamma_{h}} g_{h} \cdot \partial_{t} \tilde{u}_{h}^{\varepsilon} \\
&=\int_{0}^{T} \int_{Q_{h}} \tilde{\sigma}_{h}^{\varepsilon}: \nabla^{s} \partial_{t} \tilde{u}_{h}^{\varepsilon}=\int_{0}^{T} \int_{Q_{h}} \tilde{\sigma}_{h, \eta}^{\varepsilon}: \partial_{t}\left(C_{\varepsilon} \tilde{\sigma}_{h, \eta}^{\varepsilon}+\tilde{p}_{h, \eta}^{\varepsilon}\right) \\
&=\int_{0}^{T} \int_{Q_{h}} \frac{1}{2} \frac{d}{d t}\left(\tilde{\sigma}_{h}^{\varepsilon}:\left(C_{\varepsilon} \tilde{\sigma}_{h}^{\varepsilon}\right)+\tilde{p}_{h}^{\varepsilon}:\left(B_{\varepsilon} \tilde{p}_{h}^{\varepsilon}\right)\right) \\
&+\int_{0}^{T} \int_{Q_{h}}\left(\tilde{\sigma}_{h}^{\varepsilon}-B_{\varepsilon} \tilde{p}_{h}^{\varepsilon}\right):\left(\partial \Psi_{\varepsilon}\left(\sigma_{h, 1}^{\varepsilon}-B_{\varepsilon} p_{h, 1}^{\varepsilon}\right)-\partial \Psi_{\varepsilon}\left(\sigma_{h, 2}^{\varepsilon}-B_{\varepsilon} p_{h, 2}^{\varepsilon}\right)\right) .
\end{aligned}
$$

As the subdifferential of a convex function, the non-linear operator $\partial \Psi_{\varepsilon}$ is a monotone operator. This implies that the last integral in (B.4) is positive. We emphasize that the above calculation (e.g. the use of the fundamental theorem of calculus for $\left|\tilde{\sigma}_{h}^{\varepsilon}\right|_{C_{\varepsilon}}^{2}$ ) is justified by the regularity properties in our solution concept.

In order to exploit (B.4) further, we must make use of a trace theorem,

$$
\begin{aligned}
\left\|\tilde{u}_{h}^{\varepsilon}\right\|_{L^{2}\left(\Gamma_{h}\right)} & \leq C\left\|\tilde{u}_{h}^{\varepsilon}\right\|_{H^{1}(Q)} \leq C\left(\left\|\nabla \tilde{u}_{h}^{\varepsilon}\right\|_{L^{2}(Q)}+\left\|w_{h, \delta}\right\|_{H^{1}(Q)}\right) \\
& \leq C\left(\left\|\tilde{p}_{h}^{\varepsilon}\right\|_{L^{2}(Q)}+\left\|\tilde{\sigma}_{h}^{\varepsilon}\right\|_{L^{2}(Q)}+\left\|w_{h, \delta}\right\|_{H^{1}(Q)}\right) .
\end{aligned}
$$

With Young's inequality and Gronwall's inequality we find $\left(\tilde{u}_{h}^{\varepsilon}, \tilde{\sigma}_{h}^{\varepsilon}, \tilde{p}_{h}^{\varepsilon}\right)=(0,0,0)$, hence the uniqueness. 


\section{References}

[1] H.-D. Alber. Materials with memory, volume 1682 of Lecture Notes in Mathematics. Springer-Verlag, Berlin, 1998. Initial-boundary value problems for constitutive equations with internal variables.

[2] H.-D. Alber. Evolving microstructure and homogenization. Contin. Mech. Thermodyn., 12(4):235-286, 2000.

[3] H.-D. Alber and S. Nesenenko. Justification of homogenization in viscoplasticity: From convergence on two scales to an asymptotic solution in $L^{2}(\Omega)$. J. Multiscale Modelling, 1:223-244, 2009.

[4] D. Braess. Finite elements. Cambridge University Press, Cambridge, second edition, 2001. Theory, fast solvers, and applications in solid mechanics.

[5] A. Braides, V. Chiadò Piat, and A. Defranceschi. Homogenization of almost periodic monotone operators. Ann. Inst. H. Poincaré Anal. Non Linéaire, 9(4):399432, 1992.

[6] P. G. Ciarlet. The finite element method for elliptic problems, volume 40 of Classics in Applied Mathematics. Society for Industrial and Applied Mathematics (SIAM), Philadelphia, PA, 2002. Reprint of the 1978 original.

[7] A. Damlamian, N. Meunier, and J. Van Schaftingen. Periodic homogenization for convex functionals using Mosco convergence. Ric. Mat., 57(2):209-249, 2008.

[8] G. A. Francfort and A. Giacomini. On periodic homogenization in perfect elastoplasticity. J. Eur. Math. Soc., to appear.

[9] G. A. Francfort, F. Murat, and L. Tartar. Homogenization of monotone operators in divergence form with $x$-dependent multivalued graphs. Ann. Mat. Pura Appl. (4), 188(4):631-652, 2009.

[10] G. A. Francfort and B. Schweizer. A doubly non-linear system in small-strain visco-plasticity. Technical Report Preprint 2013-06, TU Dortmund, 2013.

[11] A. Gloria, S. Neukamm, and F. Otto. An optimal quantitative two-scale expansion in stochastic homogenization of discrete elliptic equations. ESAIM Math. Model. Numer. Anal., 48(2):325-346, 2014.

[12] K. Hackl, S. Heinz, and A. Mielke. A model for the evolution of laminates in finite-strain elastoplasticity. ZAMM Z. Angew. Math. Mech., 92(11-12):888-909, 2012 .

[13] W. Han and B. D. Reddy. Plasticity, volume 9 of Interdisciplinary Applied Mathematics. Springer-Verlag, New York, 1999. Mathematical theory and numerical analysis.

[14] M. Heida and B. Schweizer. Stochastic homogenization of a plasticity system. Technical Report (in preparation), TU Dortmund, 2014. 
[15] A. Mielke, T. Roubicek, and U. Stefanelli. T-limits and relaxations for rateindependent evolutionary problems. Calc. Var. Partial Differential Equations, 31(3):387-416, 2008.

[16] A. Mielke and A. M. Timofte. Two-scale homogenization for evolutionary variational inequalities via the energetic formulation. SIAM J. Math. Anal., 39(2):642668 (electronic), 2007.

[17] S. Nesenenko. Homogenization in viscoplasticity. SIAM J. Math. Anal., 39(1):236-262, 2007.

[18] B. Schweizer. Homogenization of the Prager model in one-dimensional plasticity. Contin. Mech. Thermodyn., 20(8):459-477, 2009.

[19] B. Schweizer and M. Veneroni. Periodic homogenization of the Prandtl-Reuss model with hardening. J. Multiscale Modelling, 2:69-106, 2010.

[20] B. Schweizer and M. Veneroni. The needle problem approach to non-periodic homogenization. NHM, 2013.

[21] B. Schweizer and M. Veneroni. Homogenization of plasticity equations with twoscale convergence methods. Applicable Analysis, 2014.

[22] M. Veneroni. Stochastic homogenization of subdifferential inclusions via scale integration. Intl. J. of Struct. Changes in Solids, 3(1):83-98, 2011.

[23] A. Visintin. On homogenization of elasto-plasticity. J. Phys.: Conf. Ser., 22:222$234,2005$.

[24] A. Visintin. Homogenization of the nonlinear Kelvin-Voigt model of viscoelasticity and of the Prager model of plasticity. Contin. Mech. Thermodyn., 18(3-4):223$252,2006$.

[25] A. Visintin. Two-scale convergence of some integral functionals. Calc. Var. Partial Differential Equations, 29(2):239-265, 2007.

[26] A. Visintin. Homogenization of the nonlinear Maxwell model of viscoelasticity and of the Prandtl-Reuss model of elastoplasticity. Proc. Roy. Soc. Edinburgh Sect. A, 138(6):1363-1401, 2008. 


\section{Preprints ab 2012/03}

2014-03 Martin Heida and Ben Schweizer

Non-periodic homogenization of infinitesimal strain plasticity equations

2014-02 Ben Schweizer

The low frequency spectrum of small Helmholtz resonators

2014-01 Tomáš Dohnal, Agnes Lamacz, Ben Schweizer

Dispersive homogenized models and coefficient formulas for waves in general periodic media

2013-16 Karl Friedrich Siburg

Almost opposite regression dependence in bivariate distributions

2013-15 Christian Palmes and Jeannette H. C. Woerner

The Gumbel test and jumps in the volatility process

2013-14 Karl Friedrich Siburg, Katharina Stehling, Pavel A. Stoimenov, Jeannette H. C. Wörner

An order for asymmetry in copulas, and implications for risk management

2013-13 Michael Voit

Product formulas for a two-parameter family of Heckman-Opdam hypergeometric functions of type $\mathrm{BC}$

2013-12 B. Schweizer and M. Veneroni

Homogenization of plasticity equations with two-scale convergence methods

2013-11 Sven Glaser

A law of large numbers for the power variation of fractional Lévy processes

2013-10 Christian Palmes and Jeannette H. C. Woerner

The Gumbel test for jumps in stochastic volatility models

2013-09 Agnes Lamacz, Stefan Neukamm and Felix Otto

Moment bounds for the corrector in stochastic homogenization of a percolation model

2013-08 Frank Klinker

Connections on Cahen-Wallach spaces

2013-07 Andreas Rätz and Matthias Röger

Symmetry breaking in a bulk-surface reaction-diffusion model for signaling networks

2013-06 Gilles Francfort and Ben Schweizer

A doubly non-linear system in small-strain visco-plasticity

2013-05 Tomáš Dohnal

Traveling solitary waves in the periodic nonlinear Schrödinger equation with finite band potentials

2013-04 Karl Friedrich Siburg, Pavel Stoimenov and Gregor N. F. Weiß

Forecasting portfolio-value-at-risk with nonparametric lower tail dependence estimates

2013-03 Martin Heida

On thermodynamics of fluid interfaces

2013-02 Martin Heida

Existence of solutions for two types of generalized versions of the Cahn-Hilliard equation 
2012-19 Martin Heida

On gradient flows of nonconvex functional in Hilbert spaces with Riemannian metric and application to Cahn-Hilliard equations

2012-18 Robert V. Kohn, Jianfeng Lu, Ben Schweizer and Michael I. Weinstein A variational perspective on cloaking by anomalous localized resonance

2012-17 Margit Rösler and Michael Voit

Olshanski spherical functions for infinite dimensional motion groups of fixed rank

2012-16 Selim Esedoğlu, Andreas Rätz, Matthias Röger

Colliding Interfaces in Old and New Diffuse-interface Approximations of Willmoreflow

2012-15 Patrick Henning, Mario Ohlberger and Ben Schweizer

An adaptive multiscale finite elment method

2012-14 Andreas Knauf, Frank Schulz, Karl Friedrich Siburg

Positive topological entropy for multi-bump magnetic fields

2012-13 Margit Rösler, Tom Koornwinder and Michael Voit

Limit transition between hypergeometric functions of type BC and Type A

2012-12 Alexander Schnurr

Generalization of the Blumenthal-Getoor index to the class of homogeneous diffusions with jumps and some applications

2012-11 Wilfried Hazod

Remarks on pseudo stable laws on contractible groups

2012-10 Waldemar Grundmann

Limit theorem for radial random walks on Euclidean spaces of high dimensions

2012-09 Martin Heida

A two-scale model of two-phase flow in porous media ranging from porespace to the macro scale

2012-08 Martin Heida

On the derivation of thermodynamically consistent boundary conditions for the CahnHilliard-Navier-Stokes system

2012-07 Michael Voit

Uniform oscillatory behavior of spherical functions $\mathrm{GL}_{\mathrm{n}} / \mathrm{U}_{\mathrm{n}}$ at the identity and a central limit theorem

2012-06 Agnes Lamacz and Ben Schweizer

Effective Maxwell equations in a geometry with flat rings of arbitrary shape

2012-05 Frank Klinker and Günter Skoruppa

Ein optimiertes Glättungsverfahren motiviert durch eine technische Fragestellung

2012-04 Patrick Henning, Mario Ohlberger and Ben Schweizer

Homogenization of the degenerate two-phase flow equations

2012-03 Andreas Rätz

A new diffuse-interface model for step flow in epitaxial growth 